\title{
The Wiring Logic of an Identified Serotonergic Neuron That Spans Sensory Networks
}

\author{
${ }^{\circledR}$ Kaylynn E. Coates, ${ }^{1}$ Steven A. Calle-Schuler, ${ }^{2}$ Levi M. Helmick, ${ }^{1}$ Victoria L. Knotts, ${ }^{1}$ Brennah N. Martik, ${ }^{1}$ \\ Farzaan Salman, ${ }^{1}$ Lauren T. Warner, ${ }^{1}$ Sophia V. Valla, ${ }^{1}{ }^{\circ}$ Davi D. Bock, ${ }^{2,3}$ and ${ }^{\circledR}$ Andrew M. Dacks ${ }^{1,4}$ \\ ${ }^{1}$ Department of Biology, West Virginia University, Morgantown, West Virginia 26506, ${ }^{2}$ Janelia Research Campus, Howard Hughes Medical \\ Institute, Ashburn, Virginia 20147, ${ }^{3}$ Department of Neurological Sciences, Larner College of Medicine, University of Vermont, Burlington, Vermont \\ 05405, and ${ }^{4}$ Department of Neuroscience, West Virginia University, Morgantown, West Virginia 26506
}

Serotonergic neurons project widely throughout the brain to modulate diverse physiological and behavioral processes. However, a single-cell resolution understanding of the connectivity of serotonergic neurons is currently lacking. Using a whole-brain EM dataset of a female Drosophila, we comprehensively determine the wiring logic of a broadly projecting serotonergic neuron (the CSDn) that spans several olfactory regions. Within the antennal lobe, the CSDn differentially innervates each glomerulus, yet surprisingly, this variability reflects a diverse set of presynaptic partners, rather than glomerulus-specific differences in synaptic output, which is predominately to local interneurons. Moreover, the CSDn has distinct connectivity relationships with specific local interneuron subtypes, suggesting that the CSDn influences distinct aspects of local network processing. Across olfactory regions, the CSDn has different patterns of connectivity, even having different connectivity with individual projection neurons that also span these regions. Whereas the CSDn targets inhibitory local neurons in the antennal lobe, the CSDn has more distributed connectivity in the LH, preferentially synapsing with principal neuron types based on transmitter content. Last, we identify individual novel synaptic partners associated with other sensory domains that provide strong, top-down input to the CSDn. Together, our study reveals the complex connectivity of serotonergic neurons, which combine the integration of local and extrinsic synaptic input in a nuanced, region-specific manner.

Key words: connectomics; Drosophila; olfaction; serotonin

Significance Statement

All sensory systems receive serotonergic modulatory input. However, a comprehensive understanding of the synaptic connectivity of individual serotonergic neurons is lacking. In this study, we use a whole-brain EM microscopy dataset to comprehensively determine the wiring logic of a broadly projecting serotonergic neuron in the olfactory system of Drosophila. Collectively, our study demonstrates, at a single-cell level, the complex connectivity of serotonergic neurons within their target networks, identifies specific cell classes heavily targeted for serotonergic modulation in the olfactory system, and reveals novel extrinsic neurons that provide strong input to this serotonergic system outside of the context of olfaction. Elucidating the connectivity logic of individual modulatory neurons provides a ground plan for the seemingly heterogeneous effects of modulatory systems.

Received Mar. 13, 2020; revised June 16, 2020; accepted June 25, 2020.

Author contributions: K.E.C., D.D.B., and A.M.D. designed research; K.E.C., S.A.C.-S., L.M.H., V.L.K., B.N.M., F.S., L.T.W., S.V.V., and A.M.D. performed research; K.E.C., L.M.H., V.L.K., B.N.M., F.S., L.T.W., and A.M.D. analyzed data; K.E.C. and A.M.D. wrote the first draft of the paper; K.E.C., S.A.C.-S., L.M.H., V.L.K., B.N.M., F.S., S.V.V., and A.M.D. edited the paper; K.E.C. and A.M.D. wrote the paper.

The authors declare no competing financial interests.

This work was supported by National Institutes of Health DC 016293 and USAFOSR FA9550-17-1-0117 to A.M.D.; Howard Hughes Medical Institute Janelia Research Campus Visiting Scientist Program project to A.M.D. and K.E.C.; and Wellcome Trust Collaborative Award 203261/Z/16/Z and National Institutes of Health RF1 MH120679 01 Award to D.D.B. The Howard Hughes Medical Institute supported the generation and hosting of the FAFB dataset. We thank the FAFB tracing community for helpful insights and contributions of neuron tracings; Tom Kazimiers and Andrew Champion for CATMAID development; Peter Li for development of the autosegmented instance of the FAFB dataset; Eric Perlman for making the autosegmented dataset available in CATMAID; the Cambridge Drosophila Connectomics group, especially Greg Jefferis, Marta Costa, Philipp Schlegel, Alex Bates, Ruairi Roberts, Robert Turnbull, Lisa
Marin, and Nik Drummond for use of hundreds of neuron reconstructions, assistance with custom analyses, and facilitating access to the autosegmented FAFB dataset; Feng Li, Mert Erginkaya, Johann Schor, Jeremy Johnson, Shrey Patel, and Nick Sweet for contributions of neuron tracings or review; Masayoshi Ito for identifying the R25C01-GAL4 line as expressing the WPN $\mathrm{N}_{B}$ s; Jay Milam for assistance with collecting confocal scans of the $\mathrm{WPN}_{\mathrm{B}}$ transmitter colocalization; Tessa Cessario for fly care; Tyler Sizemore for assistance with recombining fly stocks; Quentin Gaudry for providing the UAS-RFP, LexAop-

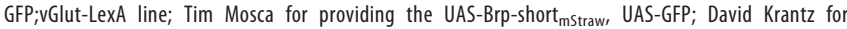
providing the hsFLP;;MCFO-1 line; Kevin Daly, Quentin Gaudry, Tim Mosca, and Tyler Sizemore for feedback on the manuscript; and Kevin Daly, Gary Marsat, and Keshav Ramachandra for insight on data analysis.

Correspondence should be addressed to Kaylynn E. Coates at kaylynn.coates@gmail.com or Andrew M. Dacks at amdacks@mail.wvu.edu.

https://doi.org/10.1523/JNEUROSCI.0552-20.2020

Copyright $\odot 2020$ the authors 


\section{Introduction}

Every neural network receives modulatory input from a variety of sources (Marder, 2012; Nadim and Bucher, 2014), and in some cases from heterogeneous populations of neurons that release the same modulatory transmitter (Schwarz and Luo, 2015; Morales and Margolis, 2017; Okaty et al., 2019). In mammals, one ubiquitous neuromodulator, serotonin, is released by thousands of widely projecting neurons that originate in the raphe nuclei (Halliday et al., 1988; Ishimura et al., 1988). Serotonergic neurons are diverse in their projections, connectivity, electrophysiological properties, and the behaviors they affect (O'Hearn and Molliver, 1984; Jensen et al., 2008; Wylie et al., 2010; Bang et al., 2012; Andrade and Haj-Dahmane, 2013; Ogawa et al., 2014; Pollak Dorocic et al., 2014; Weissbourd et al., 2014; Okaty et al., 2015; Ren et al., 2018; Sizemore et al., 2020). As a result, a significant amount of work has focused on disentangling this system (Okaty et al., 2019). Sparse reconstructions of single serotonin neurons reveal that a single serotonergic neuron can interconnect several stages within the same sensory modality (Ren et al., 2019); however, the precise patterns of connectivity of single serotonergic neurons within and across brain regions are not well understood.

The fly olfactory system is an ideal model to comprehensively explore the connectivity of individual serotonin neurons within a sensory modality. The fly olfactory system is organized similarly to vertebrates (Ache and Young, 2005), and is innervated by just one identified serotonin neuron per hemisphere. Flies detect odorants via olfactory receptor neurons (ORNs) on their antennae. ORNs expressing the same olfactory receptor converge within subcompartments, glomeruli, of the antennal lobe (AL; first-order olfactory region). Olfactory information is carried to second-order olfactory regions, including the lateral horn ( $\mathrm{LH})$ and mushroom bodies (MBs) by uniglomerular and multiglomerular projection neurons (Wilson, 2013; Grabe and Sachse, 2018; Groschner and Miesenbock, 2019). Synaptic communication between principal olfactory neurons in the AL is refined by diverse populations of local interneurons (LNs) that support many network computations (Olsen and Wilson, 2008; Root et al., 2008; Chou et al., 2010; Olsen et al., 2010; Seki et al., 2010; Yaksi and Wilson, 2010; Hong and Wilson, 2015; Nagel et al., 2015). The AL and LH also receive input from many extrinsic neurons, including two broadly projecting serotonergic neurons, the "contralaterally projecting, serotonin-immunoreactive deutocerebral neurons" (CSDns) (Dacks et al., 2006; Roy et al., 2007). The CSDns are the sole source of synaptic serotonin in the AL and LH, and receive input in both neuropils (Sun et al., 1993; X. Zhang and Gaudry, 2016; Coates et al., 2017). Furthermore, differences in synaptic connectivity between the AL and LH support compartment-specific odor coding schemes for the CSDn (X. Zhang et al., 2019). However, the organizing principles of the connectivity of this single serotonergic neuron have not been systematically studied.

To comprehensively study the connectivity of the CSDn at a single-cell resolution, we used a whole-brain EM dataset (Zheng et al., 2018) to reconstruct the CSDn in the AL and LH, identified individual synaptic partners across neuropil, and generated comprehensive connectomes of the CSDn within select AL glomeruli. While some coarse anatomic features of the CSDn are consistent across brain regions, the connectivity of the CSDn is complex, differing across glomeruli and between the AL and LH. Within the $\mathrm{AL}$, the CSDn has distinct connectivity relationships with specific LN subtypes, and interacts with unique demographics of AL neurons within each glomerulus. This pattern of organization is not conserved in the $\mathrm{LH}$, where the connectivity of CSDn varies with the transmitter content of the principal LH neurons. Finally, we demonstrate that the CSDn receives previously undescribed top-down input from three populations of extrinsic neurons. Our study reveals the complex connectivity of a single serotonergic neuron, which integrates local and extrinsic synaptic input in a nuanced, region-specific manner. This complexity at the level of a single cell likely contributes an additional degree of complexity to the role of populations of modulatory neurons.

\section{Materials and Methods}

\section{Immunocytochemistry}

Flies were raised on standard cornmeal/agar/yeast medium at $25^{\circ} \mathrm{C}$ and $60 \%$ humidity on a 12:12 light/dark cycle. The following fly stocks were used:

- MultiColor FlpOut $\left(\mathrm{MCFO}^{-1}\right)$ (Bloom, \#64085) and R14C11-GAL4 (Bloom, \#49256) (see Fig. 1A);

- MB465c-split GAL4 (Bloom, \#68371), UAS-Brp-short ${ }_{\mathrm{mStraw}}$, and UAS-GFP (Fouquet et al., 2009; Mosca and Luo, 2014) (see Fig. $1 H$ );

- R25C01-GAL4 (Bloom, \#49115), MultiColor FlpOut $\left(\mathrm{MCFO}^{-1}\right.$ ) (Bloom, \#64085), ChAT-LexA (Bloom, \#60319), vGlut-LexA (Bloom, \#60314), UAS-RFP, LexAop-GFP (Bloom, \#32229) (see Fig. 8H-K).

For immunocytochemistry, brains from approximately the same number of male and female flies were dissected in Drosophila external saline (B. Zhang et al., 2010) and fixed in $4 \%$ PFA for $30 \mathrm{~min}$ at $4^{\circ} \mathrm{C}$. Brains were then washed in PBST (PBS with 0.5\% Triton X-100), blocked for $1 \mathrm{~h}$ in 2\% BSA (Jackson ImmunoResearch Laboratories, \#001-000-162) in PBST, and incubated in primary antibodies according to Table 1 . Secondary antibodies were then applied for $24 \mathrm{~h}$. Brains were then washed, ran through an ascending glycerol series $(40 \%, 60 \%$, and $80 \%)$, and mounted in VectaShield (Vector Labs, H-1000). Images were acquired using either a $40 \times$ or $60 \times$ oil-immersion lens on an Olympus FV1000 confocal microscope. Images were processed in Olympus Fluoview FV10-ASW (RRID: SCR_014215) and Fiji (RRID:SCR_002285).

\section{EM dataset and neuron reconstruction}

The whole female adult fly brain (FAFB) EM dataset was previously generated as described previously (Zheng et al., 2018). The dataset is available for download at https://fafb.catmaid.virtualflybrain.org/ and https://www. temca2data.org. Neuron reconstructions were "traced" manually in FAFB using CATMAID (http://www.catmaid.org; RRID:SCR_006278) as previously described (Saalfeld et al., 2009; Schneider-Mizell et al., 2016). In brief, reconstructions were generated by following the confines of a neuron's cellular membrane and adding place markers (nodes). For a given neuron, all nodes are connected. Thus, users were able to reconstruct the morphology of a neuron throughout the brain volume as well as annotate synapses. A synapse was identified by the presence of (1) t-bars, (2) vesicle cloud, (3) synaptic cleft, and (4) postsynaptic density (Zheng et al., 2018). It should be noted that gap junctions cannot be visualized within the EM dataset and bulk release sites are not readily evident. All reconstructions were verified by a second, experienced tracer (Schneider-Mizell et al., 2016; Zheng et al., 2018). Some neurons were also reconstructed in part using an automated segmentation version of the FAFB dataset ( $\mathrm{Li}$ et al., 2019). These auto-traced neuron fragments were concatenated by expert users in a separate instance of FAFB and then imported and merged with previously traced fragments in the actively traced FAFB dataset using the Python tool "FAFBseg-py" (https://github.com/flyconnectome/fafbseg-py) and verified for accuracy. Finally, the PNs and LH neurons used in this analysis were published previously (Zheng et al., 2018; Dolan et al., 2019; Frechter et al., 2019; Huoviala et al., 2019; Bates et al., 2020b).

\section{CSDn reconstruction}

The left-hand CSD neuron (i.e., soma in the fly's left hemisphere) was originally identified based on the unique $\omega$-shaped projection pattern of 

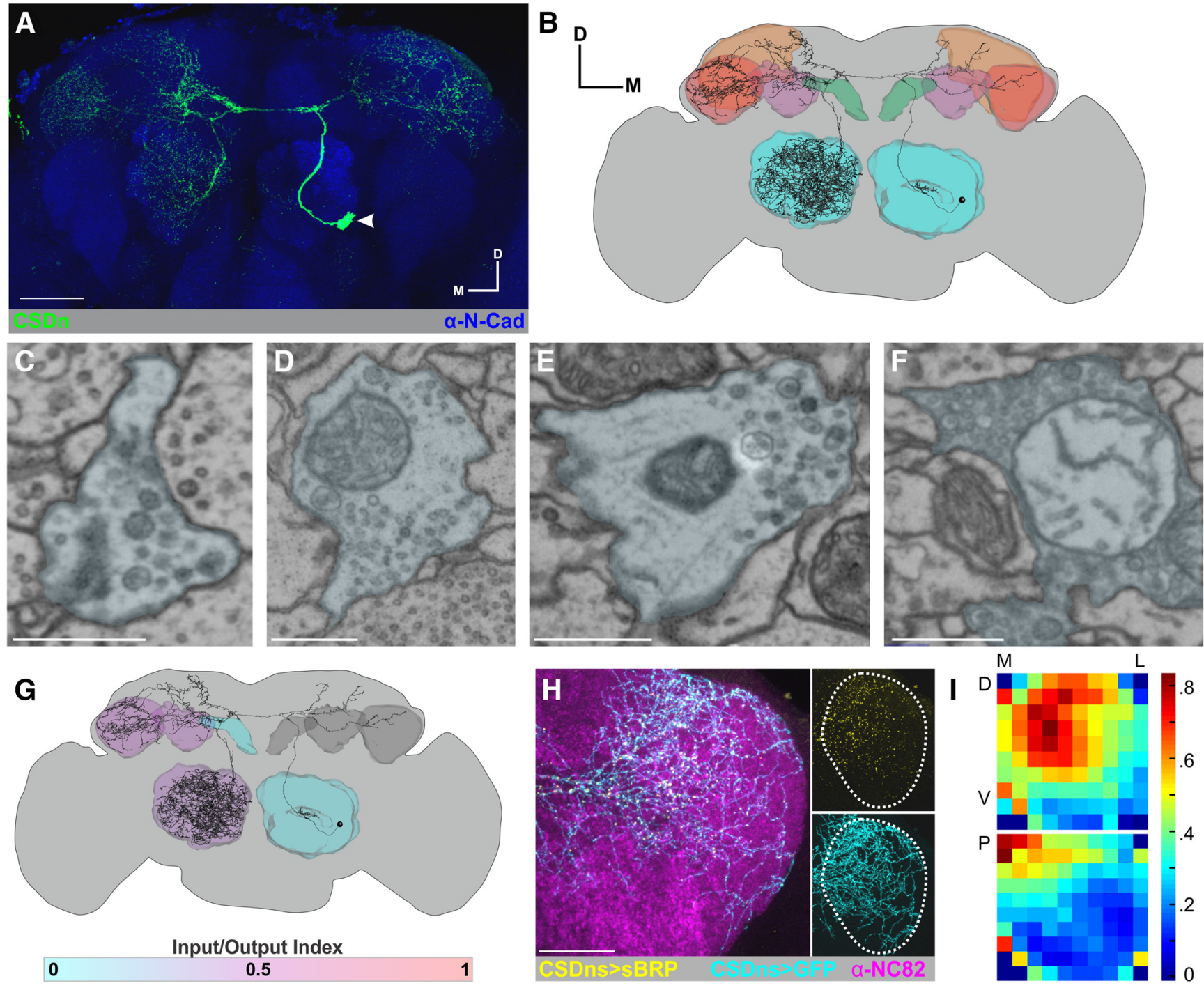

Figure 1. CSDn EM reconstruction and distribution of synaptic sites. A, MultiColor Flp0ut (green) highlights the arborization pattern of a single CSDn. N-Cadherin labeling delineates neuropil (blue). $\boldsymbol{B}$, Reconstruction of the left-hand CSDn (soma in the fly's left hemisphere) from the FAFB dataset. $\mathbf{C}-\boldsymbol{F}$, Example images of the diversity of small clear vesicles and larger dense corelike vesicles found within the CSDn branches (blue) in the AL $(\boldsymbol{C}-\boldsymbol{E})$ and LH $(\boldsymbol{F})$. Scale bars, $500 \mathrm{~nm}$. $\boldsymbol{G}$, The CSDn has a mix of input and output sites across most olfactory neuropil. Shading based on the input/output index of the CSDn was calculated as the \# inputs/(\# inputs + \# outputs) where $0=$ output only and $1=$ input only. $\boldsymbol{H}$, Expression of Brp-short puncta (yellow) labels CSDn active zones in the LH. GFP (cyan) labels the CSDn's arborizations and NC82 delineates neuropil (magenta). Scale bar, $50 \mu \mathrm{m}$. I, Heat maps of the distribution of Brp-short puncta (i.e., CSDn active zones) in the LH. Puncta density is higher in the mediodorsal and posterior regions of LH (top and bottom heat maps, respectively).

its primary process that spans the protocerebrum. The CSDn's identity was later confirmed using NBLAST (Costa et al., 2016) (RRID:SCR_015884) to query the reconstructed skeleton against a dataset of light microscopy images of single neurons (Chiang et al., 2011; Milyaev et al., 2012). The primary process of the CSDn was reconstructed from soma to ipsilateral protocerebrum to contralateral protocerebrum, to contralateral AL. Arborizations from the primary process into the LH and ALs were also reconstructed "to completion" using methods described previously (Zheng et al., 2018). For all CSDn branches traced, all presynaptic and postsynaptic sites, as well as presynaptic and postsynaptic partners were marked. In total, $23,328,903 \mathrm{~nm}$ of CSDn cable was reconstructed with 2885 presynaptic sites and 4141 postsynaptic sites marked. It should be noted that an incomplete reconstruction of the CSDn was previously published (X. Zhang et al., 2019). It should also be noted that we identified and partially reconstructed the right-hand CSDn (soma in the fly's right hemisphere; data not shown) and observed two synapses between the two CSDns.

Reconstruction of CSDn partners in 9 glomeruli

We chose 9 glomeruli to reconstruct all CSDn synaptic partners in the contralateral AL based on the lifetime sparseness of the glomerulus as well as the number of presynaptic and postsynaptic sites of the CSDn (see Fig. 2A). To reconstruct synaptic partners in specific glomeruli, the completed reconstruction of the CSDn was filtered in CATMAID so that only CSDn branches and synapses within a specific glomerulus were visible, using glomerulus volume meshes generated by Bates et al. (2020b). All neurons synapsing on the CSDn or receiving synaptic input from the CSDn were then reconstructed in a given glomerulus toward the neuron's primary neurite (i.e., backbone) and ceased when the reconstruction was sufficient to identify the neuron as a PN, LN, ORN, or other neuron type based on its soma location and/or projection pattern. Short neuron fragments that could not be reconstructed to backbone because of ambiguity of projections were deemed "orphans" and were excluded from analyses. Reconstructions were reviewed from starting synapse to the backbone (primary neurite) by a second expert tracer and then queried using NBLAST as needed.

\section{LN reconstruction and classification}

In the process of reconstructing CSDn synaptic partners in the nine select glomeruli, we found that the CSDn is synaptically connected with 84 LNs. LNs were reconstructed to the extent that, at a minimum, 
Table 1. Antibodies used for immunocytochemistry ${ }^{a}$

\begin{tabular}{lll}
\hline Antigen & Species, manufacturer, catalog \# & Incubation time (h) \\
\hline DsRed & Rabbit (Clontech, \#632496, RRID:AB_10013483) & 48 \\
GFP & Chicken (Abcam, \#ab13970, RRID:AB_300798) & $1: 250$ \\
HA-Tag & Rabbit (CST, \#3724, RRID:AB_10693385) & $1: 1000$ \\
N-Cadherin & Rat (DSHB, \#DN-Ex \#8, RRID:AB_528121) & $1: 300$ \\
RFP & Rabbit (Rockland, \#600-401-379, RRID:AB_828390) & $1: 10$ \\
AlexaFluor-488 (rabbit) & Goat (Molecular Probes, \#A-11008, RRID:AB_143165) & $1: 500$ \\
AlexaFluor-488 (chicken) & Donkey (Jackson ImmunoResearch Laboratories, \#703-545-155, RRID:AB_2340375) \\
AlexaFluor-546 (rabbit) & Donkey (Invitrogen, \#A-10040, RRID:AB_2534016) & 1:1000 \\
V5:DyLight-550 & Mouse (Bio-Rad, \#MCA1360D550GA, RRID:AB_2687576) & 24 \\
AlexaFluor-647 (rat) & Donkey (Abcam, \#ab150155, RRID:AB_2813835) & 24 \\
\hline
\end{tabular}

${ }^{a}$ Reagent information for antibodies used in Figures $1 A, H$ and $8 H-K$. For more details, see Immunocytochemistry.

allowed them to be morphologically characterized and synapses with the CSDn were marked. Two patchy LNs (2 dense all but a few [ABAF], and 1 sparse $\mathrm{ABAF}$ ) were reconstructed from soma to backbone into the finer processes of each glomerulus to synapse dense regions.

To compare LNs within and across morphologic types, K-nearest neighbor analyses (KNN) were performed between all of a neuron branchpoints and their first, third, and eighth nearest branchpoints. This generated a distribution of the distance between neighboring branchpoints for each neuron so that distributions could be compared between neurons. Similar branchpoint distributions therefore indicated that individual LNs likely belong to the same morphologic grouping. Neurons that had more branchpoints were randomly subsampled so that an equal number of branchpoints were compared across neurons. Analyses scripts and related figures were generated in $\mathrm{R}$ (https://www.r-project. org/; RRID:SCR_001905) using analysis packages within the natverse (http://natverse.org/) (Bates et al., 2020a).

CSDn active zone distribution in the $L H$

To determine the distribution of active zones in the LH, CSDn active

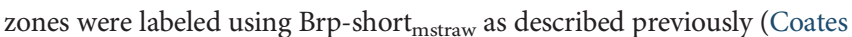
et al., 2017). Confocal $z$ stacks of the LH were acquired, and an "intensity distribution analysis" was performed using MATLAB (RRID:SCR 001622) as previously described (Lizbinski et al., 2016). In brief, the Brp puncta intensity was averaged and normalized across $10 \times 10$ bins and plotted as heatmaps.

\section{Neuronal skeleton data analysis}

Input/output index. The input/output index of presynaptic and postsynaptic sites of the CSDn was calculated by generating a list of presynaptic connectors and postsynaptic connectors within a given neuropil or glomerulus volume using PyMaid (https://github.com/schlegelp/ pymaid). Volumes used were generated by Bates et al. (2020b). For each neuropil, the input/output index was calculated as follows: \# presynaptic sites/(\# presynaptic + \# postsynaptic sites).

Other analyses. Synapse fractions, connectivity graphs, and connectivity matrices were generated using CATMAID. The volume of each neuropil mesh, branch length per glomerulus, and the number of synapses per glomerulus were calculated using PyMaid (https://github.com/ schlegelp/pymaid) and analyzed using GraphPad Prism (RRID:SCR_ 002798).

Principal component analysis (PCAs) of synapse fractions

PCAs of synapse fractions and associated analyses were performed in R using the ggfortify, ggplot, and factorextra libraries (https://rpkgs.datanovia. com/factoextra/index.html; RRID:SCR_016692). $k$-means clustering was done to determine whether points clustered in the PCA, using PC scores. (https://www.rdocumentation.org/packages/stats/versions/ 3.6.2/topics/kmeans) (MacQueen, 1967). To determine the number of clusters for the $k$-means, the silhouette method (Rousseeuw, 1987) was performed using a k-max of 4, as PC1-PC4 explained more of the variance than would be expected if the variance were equally distributed across all 9 PCs as determined by the scree plot. $k$-means clustering coloring was then applied to the PCA.

To determine whether the variability of upstream and downstream partners differed, the Euclidean distance between each downstream point with all other downstream points was measured in a pairwise manner. The Euclidean distance was determined using PC1-PC4 as explained above for the downstream partners of the 9 glomeruli and then the upstream partners of the 9 glomeruli. Statistical differences in downstream versus upstream distances were determined using a Student's $t$ test.

Experimental design and statistical analysis

Correlations, Student's $t$ tests, and descriptive statistics were calculated using GraphPad Prism version 8, and the Levene's test for homogeneity of variance was calculated using $R$.

Imaging processing and analysis

Images of the EM dataset, connectivity graphs, and reconstructions were acquired and exported from CATMAID. The rendered skeleton of the CSDn shown in Figure 1B, $C$ was generated using Blender (https://www. blender.org/; RRID:SCR_008606) and the CATMAID-to-Blender plugin (https://github.com/schlegelp/CATMAID-to-Blender). All figures were organized using CorelDrawX9 (RRID:SCR_014235).

\section{Data availability}

Neuron skeleton IDs used in this paper will be uploaded to https:// www.dacksneuroscience.com/resources.html. Neuron reconstructions generated by our group will be uploaded to the open-access website https://catmaid-fafb.virtualflybrain.org on publication.

Code accessibility

Custom scripts for analysis were generated using freely available R packages and Python tools as described above. Scripts for specific analyses are available on request.

\section{Results}

\section{General morphologic features of the CSDn are conserved across brain regions}

Serotonergic neurons, including the CSDn, have been shown to have differential innervation within olfactory brain regions (Roy et al., 2007; Gracia-Llanes et al., 2010; Singh et al., 2013; Suzuki et al., 2015; Muzerelle et al., 2016; Coates et al., 2017). However, it is unknown whether morphologic features, such as branching density and distribution of synaptic sites, vary throughout the networks that serotonergic neurons innervate. Therefore, our first goal was to systematically compare broad morphologic characteristics, such as branching density and distribution of synaptic sites of a single CSDn across and within brain regions. The somata of the two CSDns reside in the lateral cell cluster of each AL (Fig. 1A). A single CSDn produces sparse processes on its 

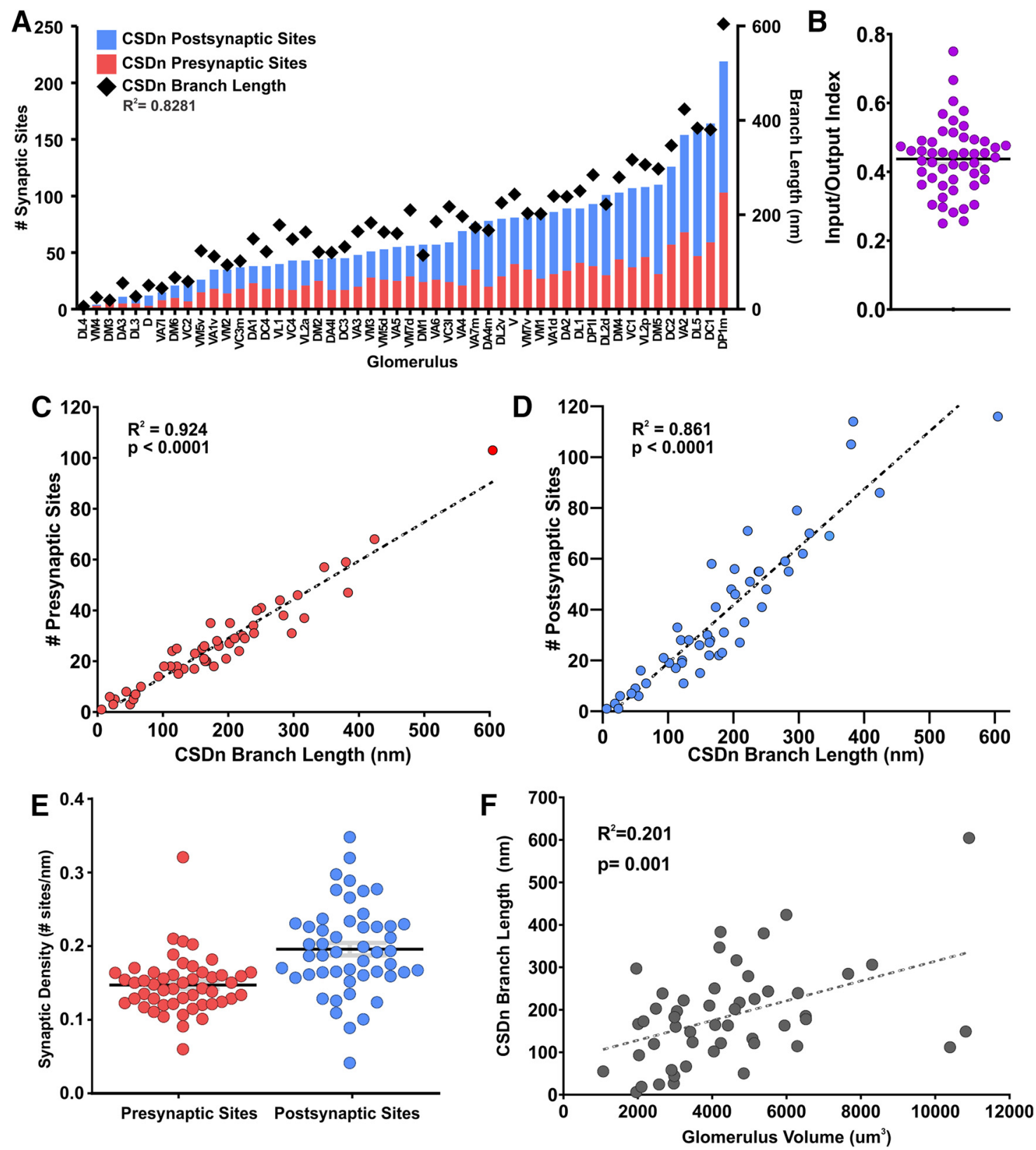

Figure 2. Glomerulus-specific innervation by the CSDn. $\boldsymbol{A}$, Glomeruli rank-ordered by the total number of CSDn synaptic sites. The number of CSDn presynaptic sites (red bars) and postsynaptic sites (blue bars) are correlated to total CSDn cable length in each glomerulus (black diamonds) $\left(R^{2}=0.8231\right)$. $\boldsymbol{B}$, Input/output index of the CSDn in each glomerulus is fairly consistent across glomeruli (mean $=0.4359$, SEM $=0.01$, coefficient of variation $=22.96 \%)$. CSDn branch length in glomeruli is highly correlated to (C) CSDn presynaptic sites $\left(R^{2}=0.924, p<0.0001\right)$ and $(D)$ number of CSDn postsynaptic sites ( $\left.R^{2}=0.861, p<0.0001\right)$. $E$, CSDn presynaptic density (red) (\# sites/nm branch length) and postsynaptic density (blue) are fairly consistent across glomeruli (coefficient of variation $=26.26 \%$ and $30.85 \%$, respectively), although postsynaptic is more distributed $(p<0.005$, Levene's test for homogeneity of variance). $\boldsymbol{F}$, CSDn branch length per glomerulus weakly correlates to glomerular volume $\left(R^{2}=0.201, p=0.001\right)$.

primary neurite within the ipsilateral AL, then follows the medial AL tract (mALT) to the ipsilateral protocerebrum where it innervates the antler, superior lateral protocerebrum, $\mathrm{MB}$ calyx (MBC), and LH. The CSDn then crosses the midline to innervate these same protocerebral regions on the contralateral side of the brain. Finally, the CSDn projects along the contralateral mALT to densely innervate the contralateral AL (Fig. $1 A, B$ ).

Because of the intricacy and size of the CSDn, we focused on reconstructing a single CSDn in the right hemisphere of the brain (i.e., CSDn soma in the fly's left hemisphere, the left-hand CSDn) where most neuronal reconstruction in the FAFB dataset (Zheng et al., 2018; Dolan et al., 2019; Frechter et al., 2019; Bates et al., 2020b) has occurred. We manually reconstructed $>2.3 \mathrm{~mm}$ of cable, which includes a complete reconstruction of the CSDn and its synaptic sites in the ipsilateral AL, contralateral AL, contralateral MBC, and contralateral LH (Fig. $1 B$ ) and confirmed our tracing was the CSDn using NBLAST (Costa et al., 2016) to geometrically compare our tracing to a skeletonized CSDn from a light microscopy image dataset (Chiang et al., 2011) (similarity score $=0.716$ ). We found that broad features of CSDn morphology and synapse distribution are similar across olfactory regions. This includes CSDn cable length per neuropil volume, which is similar in the AL $\left(35.00 \mathrm{~nm} / \mu \mathrm{m}^{3}\right)$ and $\mathrm{LH}\left(22.34 \mathrm{~nm} / \mu \mathrm{m}^{3}\right)$, respectively, despite the CSDn innervating the AL more extensively than the LH, with $13 \times 10^{6} \mathrm{~nm}(13 \mathrm{~mm})$ of cable compared with $3.6 \times 10^{6} \mathrm{~nm}(3.6 \mathrm{~mm})$ in the $\mathrm{LH}$. We did find, however, 
that the CSDn is sparser in the MBC, with a total of $0.8 \times 10^{6} \mathrm{~nm}$ of cable length and branching density of $7.48 \mathrm{~nm} / \mu \mathrm{m}^{3}$.

Expanding on previous studies using transgenic markers (Roy et al., 2007; X. Zhang and Gaudry, 2016; Coates et al., 2017; Kasture et al., 2019), we found that the CSDn has presynaptic and postsynaptic sites mixed along its neurites in all olfactory neuropil, except for the ipsilateral AL and a protocerebral region called the "antler" (Fig. 1B) in which they only have postsynaptic sites. Synaptic sites were identified by the presence of a t-bar, synaptic vesicles, synaptic vesicles in the presynaptic neuron, a synaptic cleft, and the presence of postsynaptic density on postsynaptic neurons adjacent to the synaptic cleft (Schneider-Mizell et al., 2016; Zheng et al., 2018). Serotonin can be packaged either in small clear vesicles for synaptic release or large dense-core vesicles for volume transmission (Agnati et al., 1995; Eid et al., 2013; Fuxe et al., 2015). However, while there was variability in the appearance of CSDn vesicles (Fig. $1 C-F$ ), it was not clear that any of these larger vesicles were large dense-core vesicles, although we cannot rule out the possibility of volume transmission. Whether the CSDns are capable of volume transmission is unknown; however, there is a paracrine role for $5-\mathrm{HT}$ in the AL of Drosophila, which is independent of the CSDns (X. Zhang and Gaudry, 2016; Suzuki et al., 2020).

To determine whether the ratio of presynaptic and postsynaptic sites of the CSDn differs across the contralateral AL, LH, and $\mathrm{MBC}$, we calculated an input/output index as follows: \# presynaptic sites/(\# presynaptic + \# postsynaptic sites). Surprisingly, all three neuropils have relatively similar input/output indices (ranging from 0.42 to 0.46 ; Fig. $1 G$ ) and synaptic density (ranging from 1.1 to 8 synapses $/ 10 \mu \mathrm{m}$ ). Together, this indicates that coarse traits, like total cable length and input/output index, are consistent across each olfactory brain region, despite functional differences in the organization of the AL and $\mathrm{LH}$ networks (Jeanne and Wilson, 2015; Grabe and Sachse, 2018; Jeanne et al., 2018).

The number of CSDn presynaptic sites varies between AL glomeruli, yet is consistent for a given glomerulus across animals (Coates et al., 2017). Furthermore, the distribution of the active

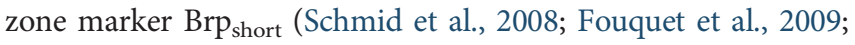
Owald et al., 2010; Mosca and Luo, 2014) is most dense in the mediodorsal and posterior regions of the LH (Fig. $1 H, I$ ). We therefore sought to determine whether the balance of presynaptic and postsynaptic sites of the CSDn in the AL varies across glomeruli or whether it is consistent as it was for global measures from the AL, LH, and MBC (Fig. $1 G$ ). We chose to focus on the AL because of its glomerular organization, which allows us to easily compare discrete subregions as opposed to the $\mathrm{LH}$ and $\mathrm{MBC}$ in which subregions are less easily discernable. We found that, while the total number of presynaptic and postsynaptic sites on the CSDn varies across glomeruli (Fig. $2 A$ ), the input/ output index for each glomerulus is fairly consistent (Fig. $2 B$; mean $=0.437, \operatorname{SEM}=0.014$, coefficient of variation $=22.96 \%$ ), and the number of presynaptic and postsynaptic sites scale with branch length for each glomerulus (Fig. 2C,D; $R^{2}=0.924$ and 0.861 for presynaptic and postsynaptic sites, respectively). We also found that the number of synaptic sites/branch length across all glomeruli is significantly less variable for presynaptic sites than postsynaptic sites (Levene's test for homogeneity of variance, $p<0.005$; Fig. $2 E$ ). Finally, we found a weak correlation between branch length and glomerular volume (Fig. 2F). Together, these results indicate that the density of CSDn presynaptic and postsynaptic sites correlates to branch length and that glomerulus-specific differences in synaptic density are because of density of innervation.

\section{CSDn connectomes across glomeruli are distinct}

The variation in the number of CSDn presynaptic and postsynaptic sites in AL glomeruli suggests that the composition of neuronal populations within each glomerulus (the "demographics") with which the CSDn interacts may also vary. Further, the glomerular demographics correlate with factors, such as the tuning breadth of ORNs and glomerular volume (Grabe et al., 2016). Specifically, glomeruli of narrowly tuned ORNs have more PNs relative to LNs and, vice versa, glomeruli of broadly tuned ORNs have more LNs than PNs. Therefore, we sought to systematically explore the differences in glomerulusspecific demographics of the CSDn's synaptic partners to determine whether there is an obvious logic behind the synaptic connectivity of the CSDn across the AL.

We reconstructed all of the CSDn's presynaptic and postsynaptic partners to identification (i.e., reconstructed to the extent that they could be identified as an ORN, LN, PN, or extrinsic neuron) within 9 glomeruli: DA1, DA2, DC1, DM1, DM5, VC2, VM1, VM2, and VM3 (Fig. 3A). These glomeruli were chosen because they vary in their odor-tuning (as measured by lifetime sparseness) (Grabe et al., 2016), number of CSDn synaptic sites, and CSDn branch length (Fig. 2), thus are likely collectively representative of AL glomeruli. Despite the large differences in glomerulus-specific innervation density of the CSDn (Fig. 2A), we found that the demographics of CSDn's downstream synaptic partners are relatively consistent across glomeruli (Fig. 3A), as the majority of the CSDn's synaptic partners are LNs regardless of odor tuning; $60 \%-94 \%$ of the CSDn's downstream synaptic partners are LNs, and lifetime sparseness does not correlate with CSDn output to LNs $\left(R^{2}=0.003, p=0.905\right.$; data not shown). For example, $>90 \%$ of the postsynaptic partners of the CSDn in DA1 and VM3 are LNs. However, DA1 has a lifetime sparseness of 0.98 and VM3 has a lifetime sparseness of 0.56 , indicating that the downstream targets do not vary with tuning breadth of a given glomerulus. However, the proportion of CSDn synapses onto ORNs varies inversely with the proportion of CSDn synapses on LNs $\left(R^{2}=0.98, p<0.001\right.$; Fig. $\left.3 F\right)$.

In contrast, the demographics of upstream synaptic partners to the CSDn in each glomerulus are highly variable (Fig. 3C). However, the neuron types that provide input to the CSDn do not correlate to glomerulus-specific CSDn innervation density or lifetime sparseness of the cognate ORNs. For example, VM2 and VM3 are both broadly tuned glomeruli (Grabe et al., 2016). However, LNs provide the largest fraction of input to the CSDn in VM2, whereas uniglomerular PNs (uPNs) provide the most input in VM3. Similarly, the CSDn receives most of its input from ORNs in both DA2 and VM1, which are narrowly tuned; yet in DA1, which is also narrowly tuned, the CSDn receives most of its input from LN subtypes (see below). To determine whether there is an obvious pattern of demographics of presynaptic partners to the CSDn, we ran a PCA. We found that the first component is largely explained by opposing vectors of vLNs and ORNs and PC2 is largely explained by opposing vectors of "uncategorized LNs" and uPNs. However, we did not find that the demographics of synaptic partners covary as their eigenvectors are largely distributed throughout the PCA (data not shown).

The high apparent degree of variability in the demography of input to the CSDn is consistent with our observation that the glomerulus-specific density of postsynaptic CSDn sites is more variable relative to the density of CSDn presynaptic sites (Fig. $2 E)$. A PCA, including all of the glomerulus-specific sets of upstream and downstream partners (Fig. 3D), confirms that the composition of upstream partners is more variable, as the mean 
A

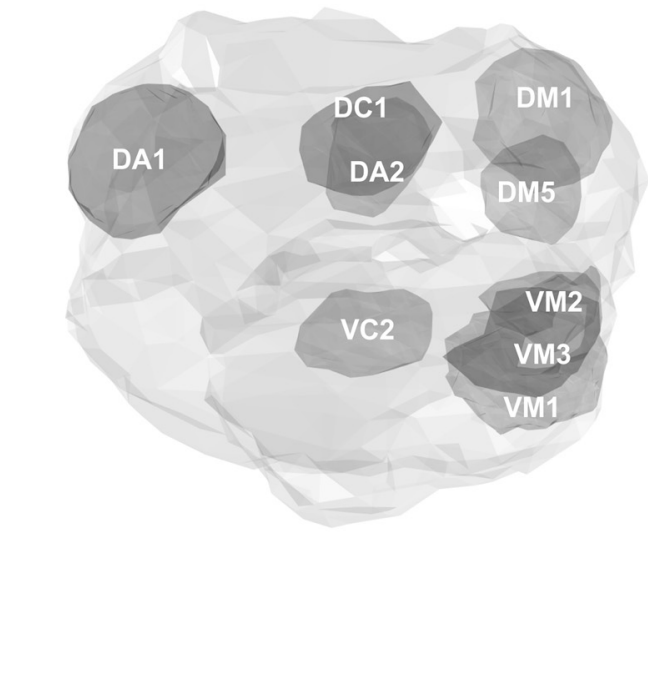

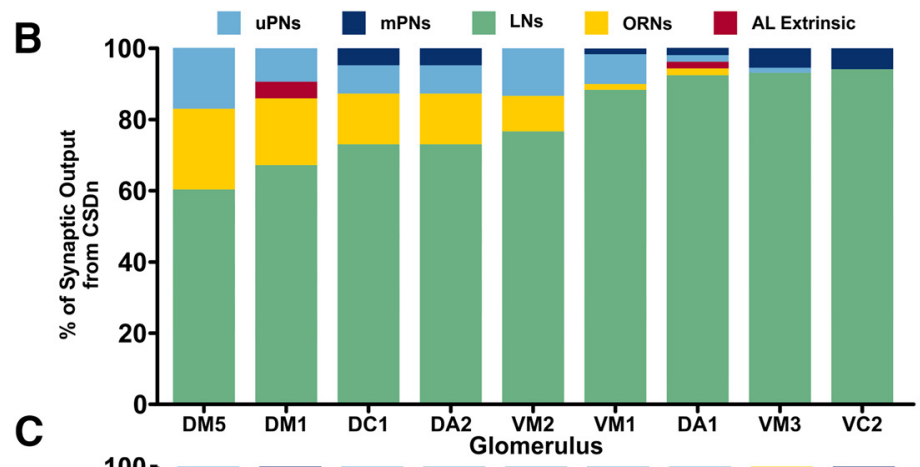

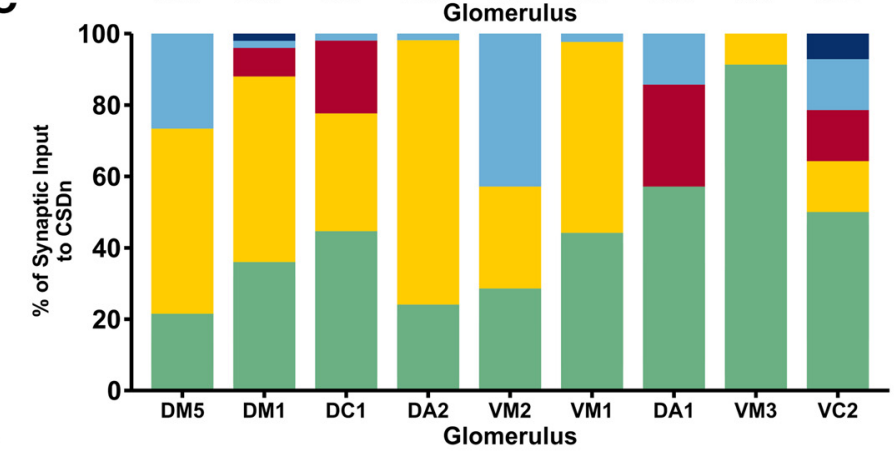

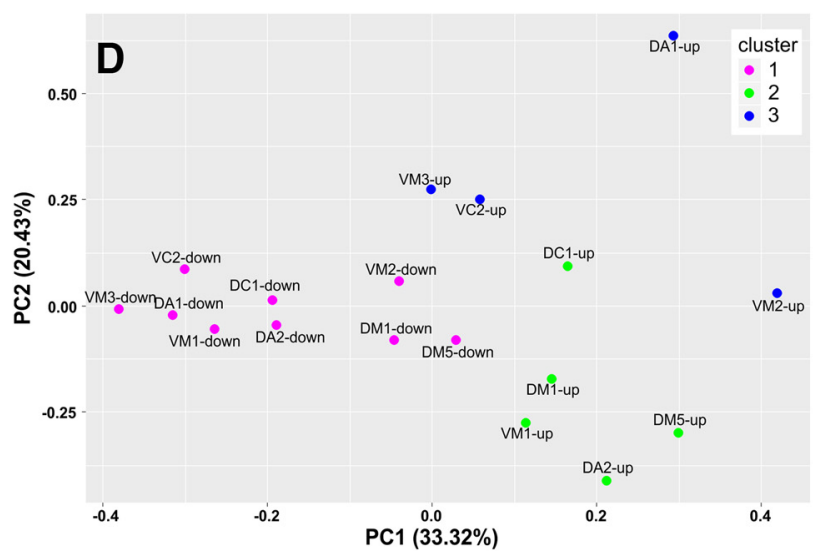

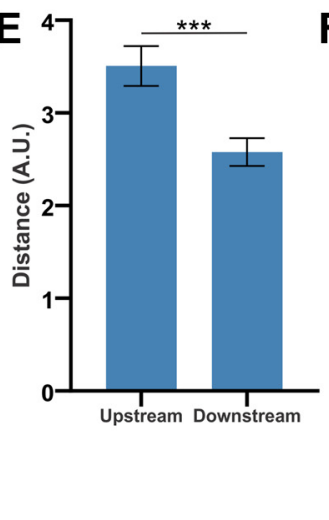

$\mathbf{F}$

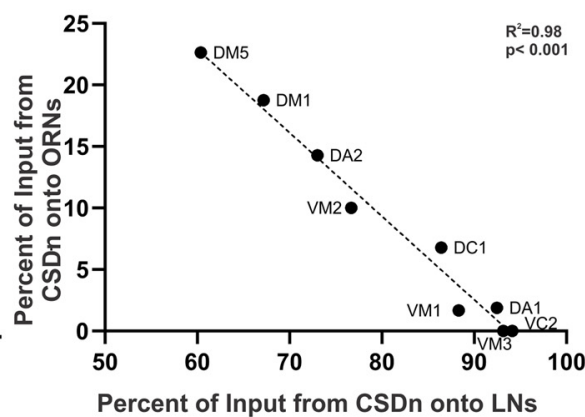

Figure 3. The CSDn has distinct connectivity across glomeruli. $\boldsymbol{A}$, Rendering of the AL highlighting glomeruli in which CSDn synaptic partners were reconstructed. $\boldsymbol{B}$, Percent of input from the CSDn onto all postsynaptic partners reconstructed in the 9 glomeruli. The CSDn predominantly targets LNs across all glomeruli. Glomerulus order based on rank-ordered amount of input from the CSDn to LNs. C, Percent of input to the CSDn from all presynaptic partners reconstructed in 9 glomeruli. Composition of presynaptic targets within each glomerulus is more diverse than for CSDn output. $\boldsymbol{D}$, Synapse fractions segregate into 3 clusters in $\mathrm{PC}$ space, based on whether the fraction is for upstream partners (magenta) or downstream partners (green and blue) based on $k$-means clustering. $\boldsymbol{E}$, The mean distance between downstream points in the PCA (D) is significantly different from the mean distance between upstream points ${ }^{* * *}, p=0.0007$, Students $t$ test). $\boldsymbol{F}$, Fraction of input from the CSDn onto LNs is inversely correlated to the percent of input from the CSDn onto ORNs.

distance between coordinates for upstream partner sets is significantly higher than between downstream partners sets (Fig. $3 E$; $p=0.0007$; Student's $t$ test). In addition, the synapse fractions of downstream partner sets cluster together separately from the upstream partner sets, which form two subclusters (Fig. 3D). This indicates that the CSDn has distinct patterns of synaptic output and input across glomeruli. Overall, while the CSDn predominantly influences LNs, glomerulus-specific input likely tempers this influence depending on the odor that the fly encounters.

\section{The CSDn has distinct connectivity with LN types}

We found that the CSDn predominantly targets LNs within the 9 glomeruli in which we reconstructed the CSDn's synaptic partners. However, there are several LN subtypes that support different network computations in the AL (Wilson and Laurent, 2005; Olsen and Wilson, 2008; Root et al., 2008; Ignell et al., 2009;
Carlsson et al., 2010; Chou et al., 2010; Olsen et al., 2010; Seki et al., 2010; Yaksi and Wilson, 2010; Das et al., 2011; W. W. Liu and Wilson, 2013; Hong and Wilson, 2015), and the manner and extent to which the CSDn interacts with each subtype are unknown. Therefore, we established a systematic framework for the connectivity of the CSDn to LNs subtypes. Based on reconstructing the synaptic partners from the 9 glomeruli, we found that the CSDn synapses with at least 84 LNs throughout the AL. Previous work has categorized LNs based on their morphology and the number of glomeruli that they innervate into the following subtypes: panglomerular, $\mathrm{ABAF}$, continuous, patchy, oligoglomerular, LNs with somata in the subesophageal zone (SEZ) and ventral (Chou et al., 2010; Das et al., 2011; Berck et al., 2016) (Fig. 4A-E). Although we did not find evidence for the CSDn being strongly connected synaptically ( $>10$ synapses) to oligoglomerular or panglomerular LNs, these LN types could be partially reconstructed neurons within the group of uncategorized 


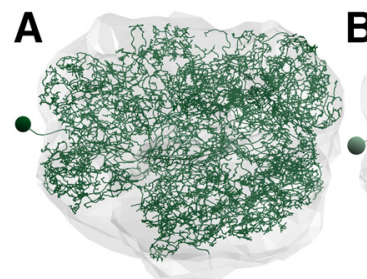

Dense ABAF

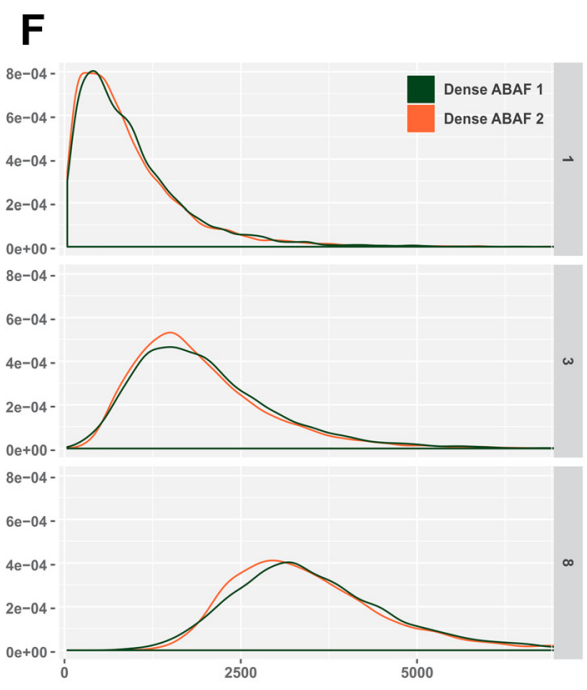

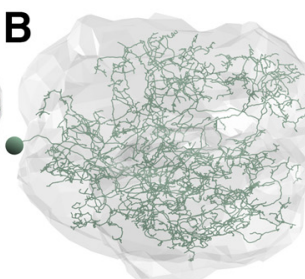

Sparse ABAF
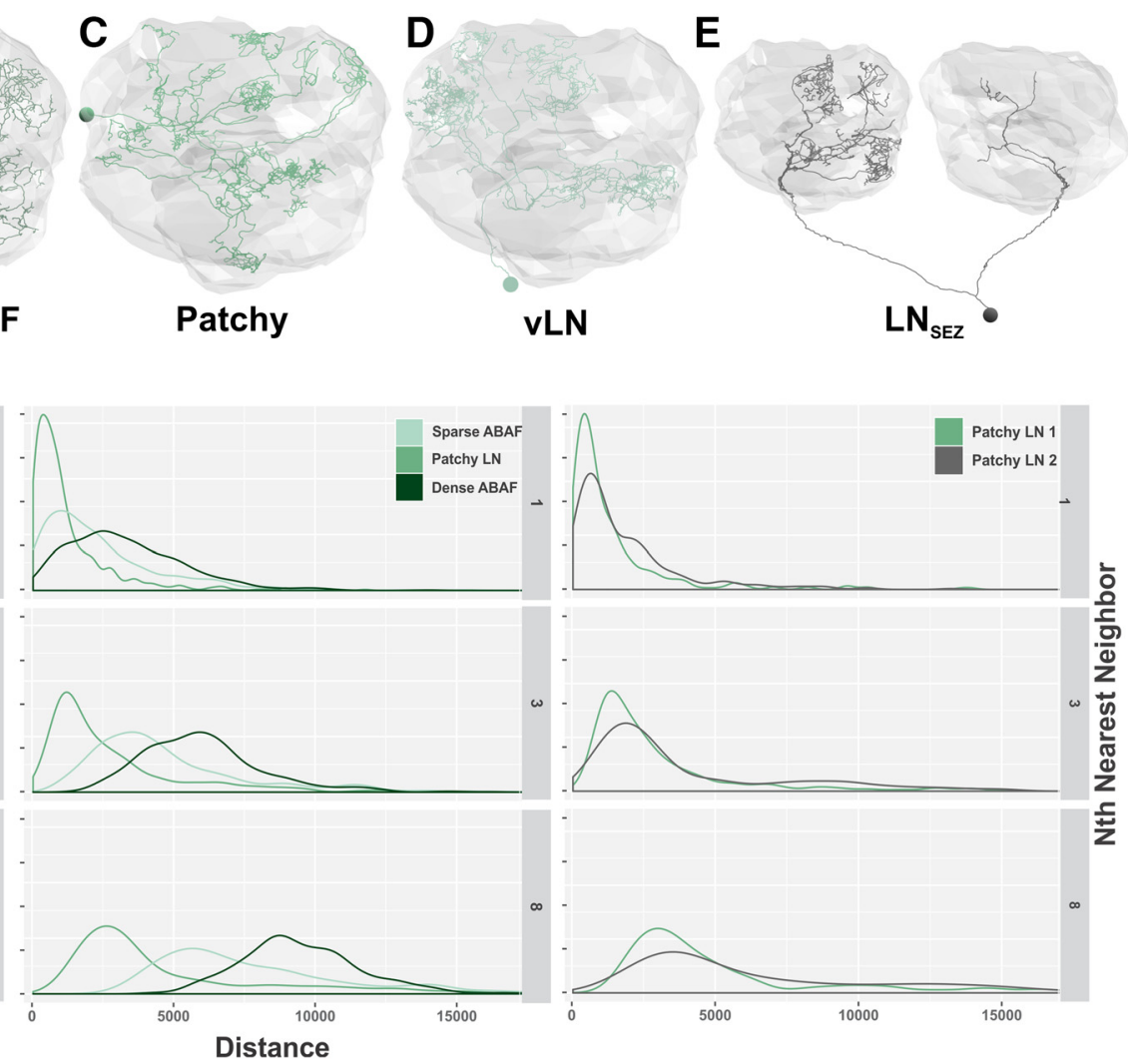

Figure 4. Classification of LNs with which the CSDn synapses. Examples of LNs with connectivity to the CSDn are as follows: $\boldsymbol{A}$, Dense ABAF LNs innervate $\sim 50$ glomeruli. $\boldsymbol{B}$, The sparse ABAF also innervates $\sim 50$ glomeruli but has far less branchpoints compared with the dense ABAF. $\boldsymbol{C}$, Patchy LNs' characteristic looping structure within the glomeruli that they innervate. $\boldsymbol{D}$, vLNs have their soma ventral to the AL and are likely glutamatergic. $E$, AL-projecting SEZ $L N s\left(L N_{S E Z}\right)$ have their somata ventral and project bilaterally to both $A L s$. These $L N s$ resemble the keystone LNs reconstructed in a larval EM dataset (Berck et al., 2016). $\boldsymbol{F}$, Distribution of KNN analyses showing the distance of the nearest neighboring branchpoints of different subclasses of LNs. Left, The distributions of the two dense ABAFs are consistent. Thus, they belong to the same morphologic class. Middle, KNN showing that sparse ABAF and dense ABAF LNs belong to two different morphologic subclasses. The patchy LN is included as an outgroup. Right, KNN distribution showing that two patchy LNs belong to the same morphologic class. Plots show distance from the first (top), third (middle), and eighth (bottom), nearest neighboring branchpoints.

LNs (see below) to which the CSDn is weakly connected but we did not classify further.

Despite the diversity of LN types, we found that $50 \%-80 \%$ of the CSDn's output to LNs is directed onto two subtypes of LNs across the 9 glomeruli; two densely branching ABAF LNs, which each innervate 48 and 49 glomeruli, respectively (data not shown), and patchy LNs, which each innervate $\sim 30$ glomeruli (Chou et al., 2010). The CSDn provides $>600$ synapses to the two dense ABAF LNs, approximately one-third of its synaptic output within the $\mathrm{AL}$ and receives input from the ABAFs at $>50$ synaptic sites (Fig. $5 A-C$ ). We identified and reconstructed another ABAF LN, which innervates at least 44 glomeruli but branches far less extensively than the two densely branching ABAF LNs. Using a KNN, we calculated the distribution of distances between branchpoints of a neuron and its neighboring branchpoints (see Materials and Methods). We found that the branchpoint distributions of the two dense ABAF LNs closely overlap, in contrast to the sparse ABAF LN, which has a much larger range of branchpoint distributions and thus has far less overlap with the dense ABAF (Fig. $4 F$ ). This indicates that the dense and sparse ABAF LNs indeed represent two separate subclasses. Moreover, this sparse ABAF LN has little connectivity to the CSDn.

The CSDn is also highly connected to the patchy LNs, which innervate subvolumes, or "patches," of individual glomeruli and, as a population, tile across the entirety of the AL (Chou et al., 2010). Before innervating a given glomerulus, patchy LNs have long, highly looping processes that lack presynaptic or postsynaptic sites, which further assisted in their identification. Although the biological significance of these convoluted processes is unclear, similar examples have been reported in Drosophila and other invertebrates (Otopalik et al., 2017a,b; Tobin et al., 2017). Once within a glomerulus, a patchy LN branches extensively within a subregion of a glomerulus and produces many presynaptic and postsynaptic sites (Fig. 4C) (Chou et al., 2010). In contrast to the dense ABAF, the CSDn has 2:1 reciprocal connectivity to a population of at least 21 patchy LNs (Fig. 5C). KNN analysis supports the hypothesis that these patchy LNs belong to the same morphologic subclass (Fig. $4 F$ ). For the remaining LNs, the CSDn has weak ( $<5$ synapses) reciprocal synaptic connectivity with ventral LNs (Figs. $4 D, 5 A, B$ ), which are most likely glutamatergic (Das et al., 2011; W. W. Liu and Wilson, 2013), as well as a pair of bilaterally projecting LNs whose somas are in the SEZ (AL-projecting SEZ LNs; $\mathrm{LN}_{\mathrm{SEZ}}$ ), similar to the "Keystone" LNs (Fig. 4E) on which the CSDn synapse in the larval Drosophila AL (Berck et al., 2016). An additional 46 unclassified LNs, which were reconstructed from the 9 glomeruli, are grouped as "uncategorized LNs" as they are not classified as ABAF, $\mathrm{LN}_{\mathrm{SEZ}} \mathrm{s}$, patchy, or vLNs (Fig. $5 C$ ). Collectively, the CSDn provides at least 212 synapses to and receives at least 148 synapses from LNs that are not ABAFs or patchy LNs, although they are likely not a monolithic group. Together, these results suggest that, while LNs are the major target of the CSDn in the AL, the CSDn preferentially targets one 

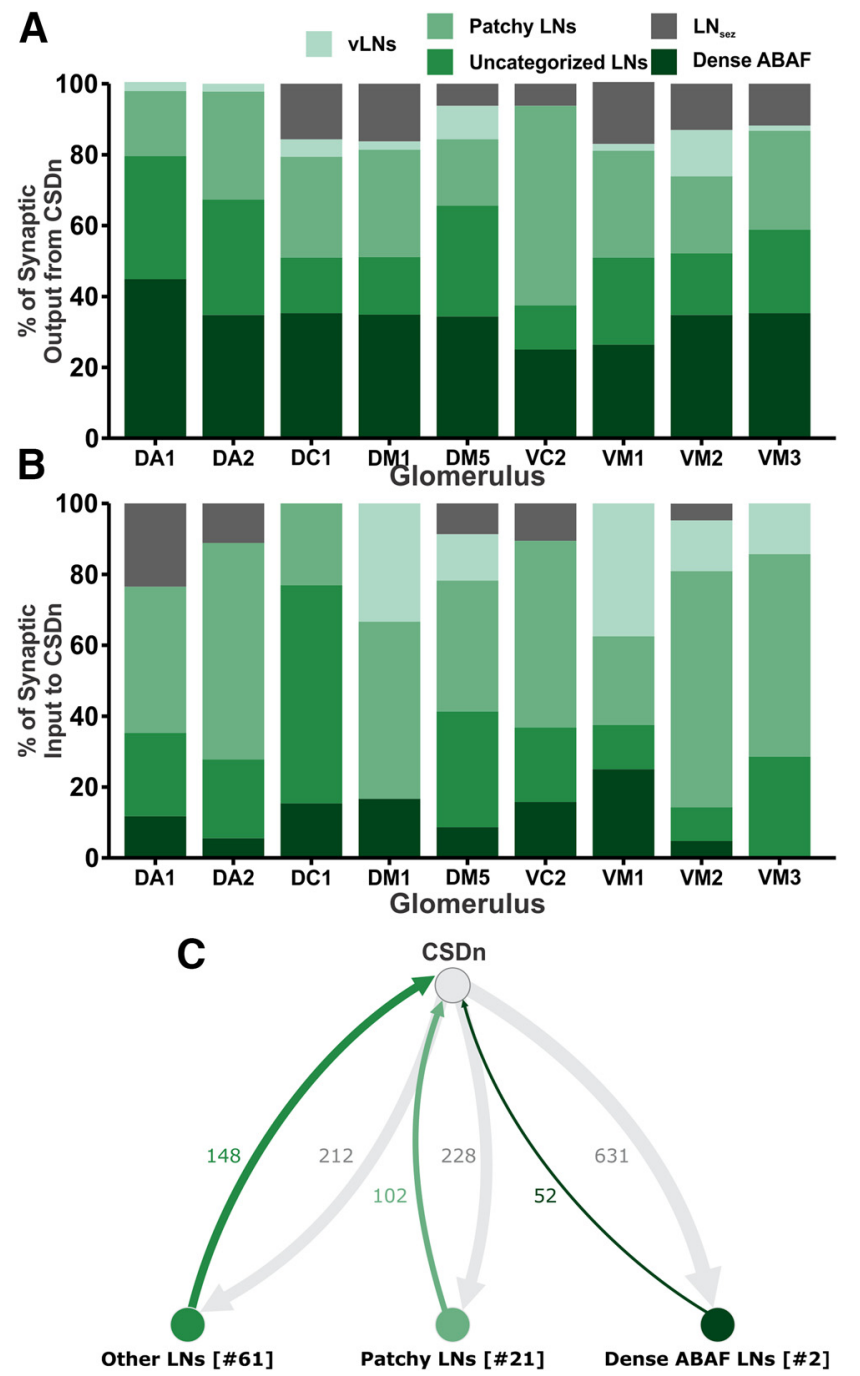

Figure 5. The CSDn has distinct connectivity with $L N$ subpopulations. $A$, Percent of synaptic input from the CSDn onto LN subtypes. The CSDn provides input to most LN subtypes across all 9 glomeruli, except for $\mathrm{LN}_{\mathrm{SEZ}}$. Dense ABAFs and patchy $\mathrm{LNs}$ appear to be the main LN type the CSDn targets regardless of glomerulus identity. $\boldsymbol{B}$, The CSDn receives far more of its LN synaptic input from patchy LNs across 9 glomeruli. C, The CSDn is overall most strongly connected to two dense ABAF LNs as well as patchy LNs. "Other LNs" include $L N_{\text {SEZ }}$, vLNs, and otherwise uncategorized LNs. \#, number of neurons within a population. Synaptic connections derive from the 9 completely reconstructed glomeruli in Figure 3 and synaptic connections from other glomeruli found in the course of reconstructing the $\mathrm{LN}$.

particular LN subclass, the dense ABAF LNs, and has reciprocal connectivity with the larger population of patchy LNs (Fig. $5 C$ ).

CSDn connectivity varies across olfactory processing regions Individual serotonin neurons in mice and flies alike span several stages of olfactory processing (Dacks et al., 2006; Coates et al., 2017; Ren et al., 2019); however, it is unclear whether the wiring logic of individual serotonergic neurons is conserved across brain regions. For instance, do serotonergic neurons interact with the same individual neuron across multiple brain regions? Are general connectivity rules maintained from one processing stage to the next? We began by asking whether the connectivity of the CSDn to individual PNs differs across the AL and $\mathrm{LH}$ as both the CSDn and PNs span both brain regions. Previously published PN reconstructions (Zheng et al., 2018; Bates et al., 2020b; Marin et al., 2020) were grouped into three subtypes based on whether they were uPN or multiglomerular $(\mathrm{mPN})$, and the AL tract along which the mPNs project, either mALT or mlALT (Bates et al., 2020b). In addition, mPNs also differ functionally in terms of the transmitter they express, with mALT mPNs being cholinergic and mlALT mPNs being GABAergic (Liang et al., 2013; Parnas et al., 2013; Strutz et al., 2014; Bates et al., 2020b). Collectively, 78 mALT uPNs (of 149), 46 mALT mPNs, and 28 mlALT mPNs (of 197 total mPNs) are synaptically connected to the CSDn across the AL and LH (Fig. 6A-C). This is likely an underestimation of the total CSDn:PN connectivity in the AL, as not all PN dendrites were reconstructed to completion. However, any PN branches that synapse with the CSDn in the 9 glomeruli that we sampled were reconstructed to the primary process of the neuron to the extent which the neuron could be identified as a PN (i.e., to identification).

We found that the three PN types have distinct connectivity with the CSDn from one another both within and across the AL and LH. For instance, of the different PN subtypes, the CSDn is most strongly connected to the uPNs as a population, maintaining reciprocal connectivity with 3:2 uPN:CSDn ratio in both the $\mathrm{AL}$ and $\mathrm{LH}$ (Fig. $6 D, D^{\prime}$ ). While most individual uPNs are weakly connected to the CSDn ( $<10$ synapses), a few individual uPNs are strongly connected. In particular, two DM5 uPNs have 16-18 synapses onto the CSDn in the $\mathrm{AL}$, consistent with previous work using transgenic and physiological approaches demonstrating that DM5 PNs synapse with the CSDn (Coates et al., 2017). The mALT mPNs also have balanced reciprocal synaptic connectivity with the CSDn in the AL; however, they have almost no connectivity with the CSDn in the LH (Fig. $6 D, D^{\prime}$ ). Finally, the CSDn has unidirectional connectivity with the mlALT mPNs in the AL with the CSDn providing input to, but not receiving input from, them (Fig. 6D). Thus, like LNs, the connectivity of the CSDn with PNs varies across PN subtypes and varies between the AL and the LH. Even within a given glomerulus, individual uPNs have different connectivity with the CSDn. For example, of the five DA2 uPNs, one synapsed on the CSDn in the AL, two received synaptic input from the CSDn in the AL, one is reciprocally connected to the CSDn in the LH, and a fifth receives input from the CSDn in both brain regions (data not shown). The uPNs from the same glomerulus can differ in their connectivity within the AL (Tobin et al., 2017) and MBC (Caron et al., 2013), and it appears that this feature extends to the CSDn as well.

Does an individual serotonin neuron maintain its connectivity to individual neurons across brain regions? We asked whether the CSDn is synaptically connected to the same individual uPNs in both the AL and LH. Although the CSDn could be connected to a given $\mathrm{PN}$ within both the $\mathrm{AL}$ and the $\mathrm{LH}$, the connectivity relationship of a given $\mathrm{uPN}$ is rarely conserved across these processing stages (Fig. 6E). For instance, in the AL, there are $21 \mathrm{uPNs}$ that receive synaptic input from the CSDn but are not reciprocally connected. Of these 21 uPNs, only 2 maintain this relationship in the LH. Thus, the CSDn has heterogeneous synaptic connectivity to individual neurons across processing stages of the olfactory system. Furthermore, a single serotonergic neuron can be differentially connected to "equivalent" neurons within (i.e., uPNs from the same glomerulus) and between multiple processing stages.

Finally, we sought to determine whether broad features of CSDn connectivity to principal neuron types in the AL are maintained for the principal neuron types of the LH. While the CSDn primarily targets LNs within the AL, it has extensive connectivity with a diverse set of neurons within the LH in addition to PNs. Although we did not comprehensively reconstruct all of the synaptic partners of the CSDns within the LH, we were able to 

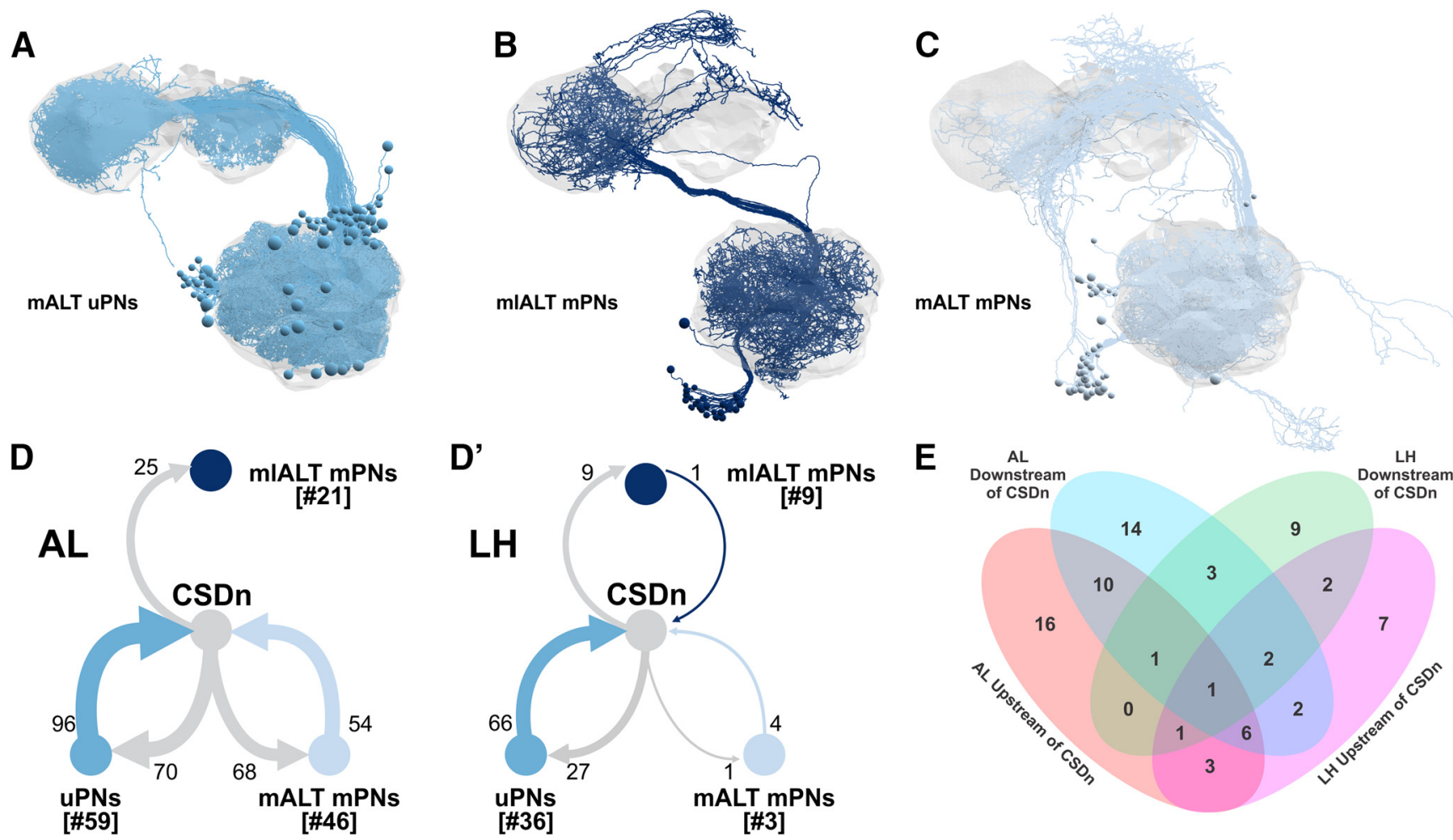

Figure 6. CSDn connectivity with PNs in the AL and LH. EM reconstructions of PNs with connectivity to the CSDn $(\boldsymbol{A}) \mathrm{mALT}$ uPNs, $(\boldsymbol{B}) \mathrm{mIALT} \mathrm{mPNs}$, and $(\boldsymbol{C}) \mathrm{mALT}$ mPNs. Number of synaptic connections of PN subtypes with the CSDn in the AL $(\boldsymbol{D})$ and $L H\left(\boldsymbol{D}^{\prime}\right)$. $\boldsymbol{E}$, uPNs have varied connectivity with the CSDn across the AL and LH. Number indicates number of neurons, rather than synapse counts.

leverage a rich dataset of previously traced neurons (Dolan et al., 2019; Frechter et al., 2019; Bates et al., 2020b) to subsample the populations of cells to which the CSDns are connected. Additionally, we included the connectivity of right-hand CSDn, which was partially reconstructed in the protocerebrum. There are at least 82 cell types of LH neurons (Dolan et al., 2019), which can be classified into three broad anatomic types; LH output neurons (LHONs), LH local neurons (LHLNs), and LH input neurons (LHINs). These can be further subdivided based on cell body cluster location (anterior ventral; $\mathrm{AV}$, anterior dorsal; $\mathrm{AD}$, posterior ventral; PV and posterior dorsal; PD) and their expression of acetylcholine, GABA, or glutamate (Dolan et al., 2019; Frechter et al., 2019; Bates et al., 2020b). Both CSDns are synaptically connected to neurons from all three categories (Fig. 7A-D); and the strength of specific relationships, as well as the degree to which each relationship is symmetrical, varies with putative transmitter. The CSDns are most strongly reciprocally connected to cholinergic LHONs, GABAergic LHLNs, and glutamatergic LHLNs (Fig. 7E, $F)$. Although the CSDns have mostly sparse (between 1 and 10 synapses) connectivity to individual LH neurons, the CSDns are strongly connected to individual LHINs, receiving strong synaptic input from two cholinergic LHINs and providing strong synaptic input to a GABAergic LHIN (Fig. 7C,E,F). Thus, the general connectivity logic of the CSDn appears to differ between the AL and $\mathrm{LH}$. While the CSDn connectivity in the AL is highly biased toward LNs, it has more distributed connectivity across principal cell types in the LH, with some exceptions, such as individual LHINs.

The CSDn receives abundant synaptic input from distinct populations of protocerebral neurons

While CSDn processes have a mixture of presynaptic and postsynaptic sites in olfactory neuropils, the CSDn only receives synaptic input along its main process as it passes through a protocerebral region called the antler (Fig. 8A). By reconstructing neurons that provide synaptic input to the CSDns in this region, we identified a population of 10 morphologically similar neurons that collectively provide $>240$ synapses to both CSDns in the antler, as well as the $\mathrm{LH}$, and several other protocerebral regions (Fig. $8 B$ ). These neurons have their somata near the $\mathrm{LH}$, project ventrally into the ipsilateral wedge where they branch extensively before projecting back dorsally, crossing the midline and then projecting ventrally into the contralateral wedge. The descending processes in each hemisphere project anteriorly into several mechanosensory neuropils (e.g., the anterior ventrolateral protocerebrum and saddle). These wedge projection neurons (WPNs) are morphologically similar to a population of unilaterally projecting WPNs (Suver et al., 2019) and have also been identified in prior analyses of clonal units (Ito et al., 2013). Because of their projections to both brain hemispheres, we refer to these WPNs as "bilaterally projecting WPNs" $\left(\mathrm{WPN}_{\mathrm{B}} \mathrm{s}\right)$. Consistent with a role in processing mechanosensory input to the antennae, the $\mathrm{WPN}_{\mathrm{B}} \mathrm{S}$ receive a large amount of synaptic input within the lateral portion of the ipsilateral wedge, a second-order mechanosensory center (Kamikouchi et al., 2009; Lai et al., 2012; Vaughan et al., 2014; Matsuo et al., 2016; Patella and Wilson, 2018; Suver et al., 2019). It should be noted that there are $>10 \mathrm{WPN}_{\mathrm{B}} \mathrm{s}$ in total as we did identify other $\mathrm{WPN}_{\mathrm{B}} \mathrm{S}$ that did not provide synaptic input to the CSDns (data not shown).

The $\mathrm{WPN}_{\mathrm{B}} \mathrm{S}$ form a three-tiered feedforward network in which subsets of $\mathrm{WPN}_{\mathrm{B}} \mathrm{s}$ provide synaptic input to $\mathrm{WPN}_{\mathrm{B}} \mathrm{S}$ in the next tiers, but the subsequent tiers provide little, if any, feedback to the $\mathrm{WPN}_{\mathrm{B}} \mathrm{s}$ from which they receive input (Fig. $8 F$ ). We further classified the $\mathrm{WPN}_{\mathrm{B}} \mathrm{s}$ based on their position within this connectivity network as $\mathrm{WPN}_{\mathrm{B}} 1$ (Fig. 8 C; two neurons), $\mathrm{WPN}_{\mathrm{B}} 2$ 

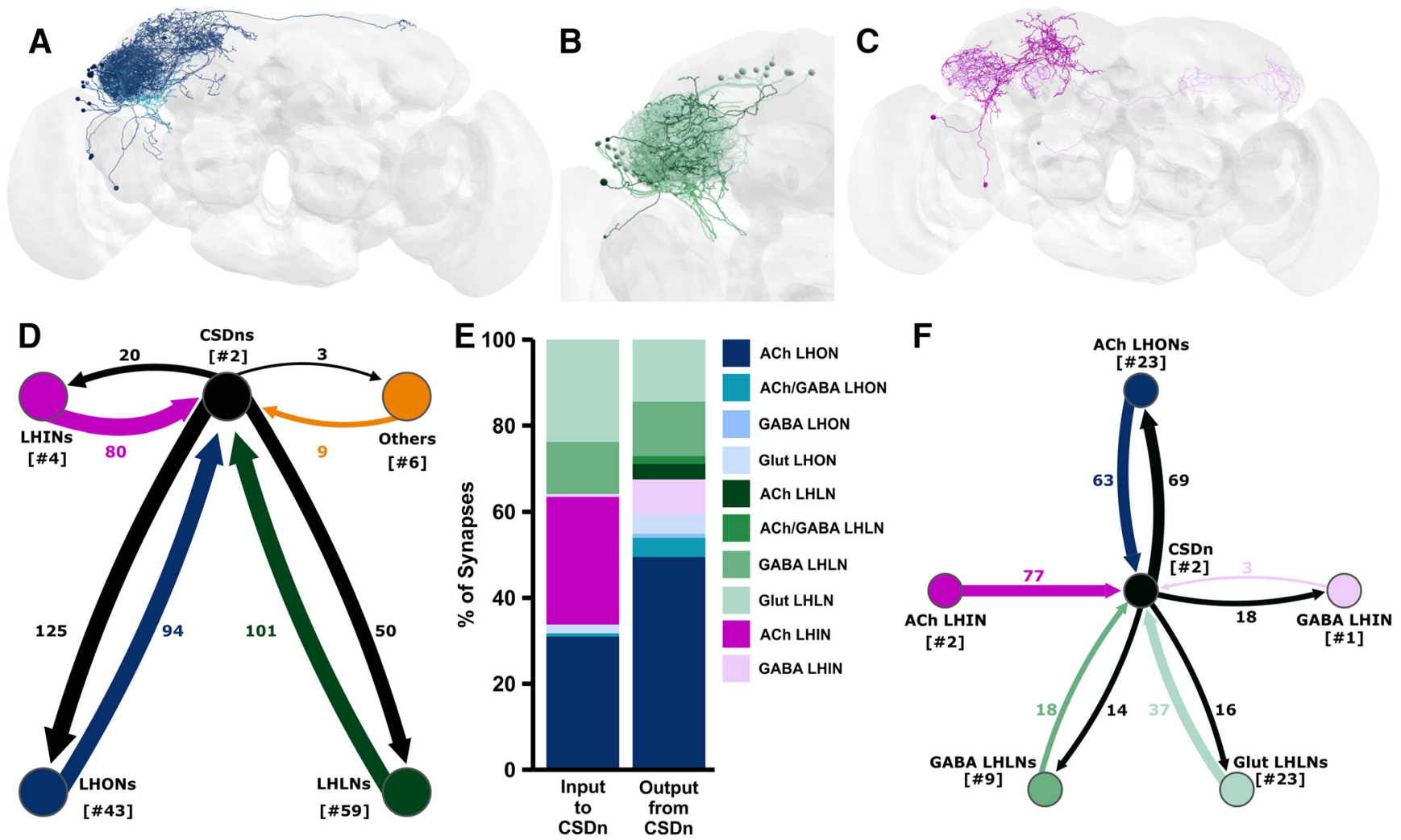

Figure 7. CSDn connectivity with LH neurons. EM reconstruction of $(\boldsymbol{A})$ LHONs, $(\boldsymbol{B})$ LHLNs, and $(\boldsymbol{C})$ LHINs that have connectivity with both CSDns. Colorization based on transmitter content (see $\boldsymbol{E}$ ). D, The CSDn reciprocally connectivity to each class of LH neurons. $\boldsymbol{E}$, Percent of input to and from the CSDns onto populations of LH neurons. The CSDns have the most connectivity to and from cholinergic LHONs followed by glutamatergic and GABAergic LHLNs. $\boldsymbol{F}$, Connectivity of LH neuron types that are strongly connected based on transmitter content.

(Fig. $8 D$; two neurons), and $\mathrm{WPN}_{\mathrm{B}} 3$ (Fig. $8 E$; six neurons). The $\mathrm{WPN}_{\mathrm{B}} 1 \mathrm{~s}$ are morphologically distinct from $\mathrm{WPN}_{\mathrm{B}} 2$ and $\mathrm{WPN}_{\mathrm{B}} 3$ neurons as they have an additional anterodorsal projecting branch that extends from the medial saddle branch and their cell bodies are larger than the other $\mathrm{WPN}_{\mathrm{B}} \mathrm{s}$ (Fig. $8 \mathrm{C}$ ). Using the MultiColor FlpOut technique (Nern et al., 2015), we identified a GAL4 line that is expressed by both $\mathrm{WPN}_{\mathrm{B}} 1 \mathrm{~s}$ and a subset of the $\mathrm{WPN}_{\mathrm{B}} 2 / 3 \mathrm{~s}$ (Fig. $8 H, I$ ). We then combined this GAL4 with either a Cha ${ }^{\text {MI04508 }}$-LexA::QFAD or a VGlut ${ }^{\text {MI04979 }}$-LexA::QFAD protein trap line (Diao et al., 2015) and found that many of the $\mathrm{WPN}_{\mathrm{B}} \mathrm{S}$ in this GAL4 line are cholinergic, but none is glutamatergic (Fig. 8J,K). The $\mathrm{WPN}_{\mathrm{B}} \mathrm{S}$ have weak, nondirectionalityselective wind responses (Suver et al., 2019), suggesting that these neurons could be relaying mechanosensory information to the CSDns. In the process of reconstructing the $\mathrm{WPN}_{\mathrm{B}} \mathrm{s}$, we also identified an additional unilaterally projecting protocerebral neuron, which provides at least 90 synapses to both CSDns in the antler, superior medial protocerebrum, and superior lateral protocerebrum (Fig. 9).

Finally, we identified a set of four protocerebral neurons that project into the AL where they synapse extensively on the CSDn. These neurons have their somata along the dorsal midline of the brain, project laterally into the superior medial and intermediate protocerebra and ventrally, with one branch extending into the SEZ and another into the ipsilateral AL (Fig. 10A). Based on their projections, we refer to these neurons as the SIMPAL (superior intermediate/medial protocerebra to $\mathrm{AL}$ ) neurons. The SIMPAL neurons innervate $\sim 23$ glomeruli before crossing the antennal commissure into the contralateral AL (Fig. 10). Collectively, these extrinsic neurons provide at least 188 synapses to the lefthand CSDn (Fig. 10C). Although the role of the SIMPAL neurons is not currently known, interestingly, they provide their greatest input to the CSDn in glomeruli that are tuned to attractive food odors, including limonene (DC1) (Dweck et al., 2013), acetic acid (DP1l) (Prieto-Godino et al., 2017), apple cider vinegar (DP1m, VA2) (Semmelhack and Wang, 2009), 2,3 butanedione (VA2) (Semmelhack and Wang, 2009; Knaden et al., 2012), ethyl lactate (VC4) (Fuyama, 1976), and 2-oxovaleric acid (VL2p) (Silbering et al., 2011; Huoviala et al., 2019). In particular, the SIMPAL neurons were strongly connected to the CSDn in DP1m, DP1l, and DC1, with 10-25 synapses in each (data not shown). This suggests that the influence of the SIMPAL neurons on the CSDn is nonuniform, localized, and potentially within the context of food attraction. We also found that the SIMPAL neurons receive significant input from the dense ABAF LNs (Fig. $10 D$ ) and most strongly in the same glomeruli in which the SIMPAL neurons provide the greatest synaptic input to the CSDn (Fig. 10E). This suggests that the CSDn and the SIMPAL neurons are reciprocally connected, although any influence of the CSDn on the SIMPAL neurons would be polysynaptic via the ABAF LNs.

\section{Replication of connectivity principles across animals}

While this manuscript was in preparation, a second dense reconstruction of a portion of a Drosophila central brain (called the "Hemibrain") was made publicly available (Scheffer et al., 2020). Many of the synaptic partners and connectivity relationships we found in the FAFB dataset were confirmed by the hemibrain dataset. We have included body IDs (Table 2) of neuron types in the Hemibrain that we describe from FAFB, although this list is not comprehensive. Thus, the broad patterns of connectivity that we describe for the CSDn are likely conserved across individual animals. 


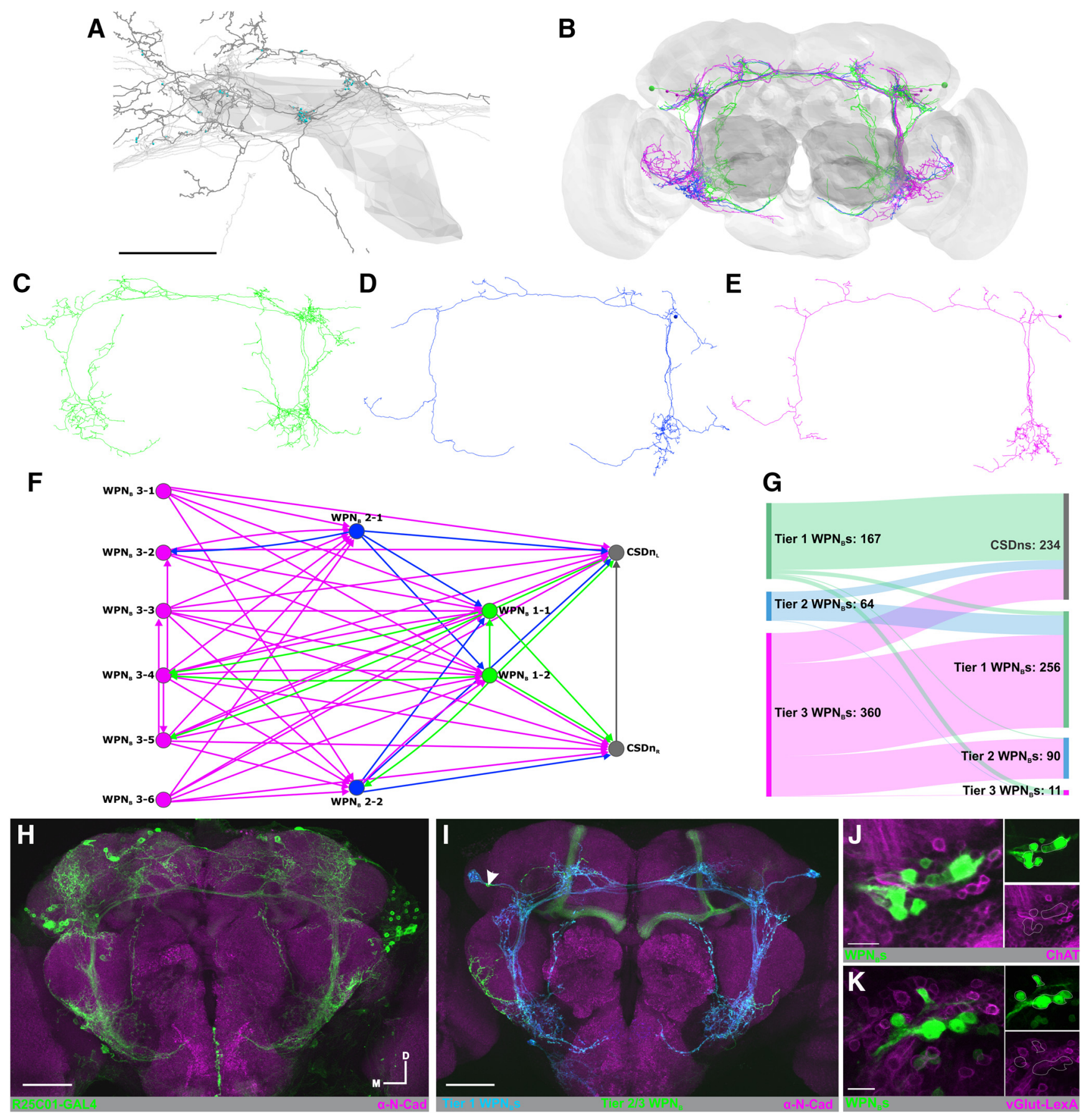

Figure 8. $W \mathrm{WPN}_{B} S$ provide top-down input to the $C S D n . A, W P N_{B} S$ (light gray) provide input to the CSDn (dark gray) in the antler. Cyan represents CSDn postsynaptic site markers. $\boldsymbol{B}$, EM reconstructions of $12 \mathrm{WPN}_{B}$ s that provide a top-down input to the $C S D n$ in the antler and portions of the protocerebrum. EM reconstructions of a Tier 1 WPN $N_{B}\left(\boldsymbol{C} ; g^{2}\right.$ reen), Tier 2 WPN ${ }_{B}(\boldsymbol{D} ;$ magenta), and Tier $3 W^{W} N_{B}\left(E ;\right.$ blue). $\boldsymbol{F}$, The WPN ${ }_{B} S$ provide input to the $C S D n s$ in a three-tiered, feedforward network where the Tier 1 WPN $S$ (green) are morphologically distinct from Tier 2 (blue) and Tier 3 (magenta). G, Tier $3 \mathrm{WPN}_{B}$ s provide strong input to the Tier 1 and Tier 2 WPN $_{B}$ s as well as the CSDn. $\boldsymbol{H}$, R25C01-driven expression of GFP (green) includes populations of $W_{\mathrm{WPN}} \mathrm{S}$. I, MultiColor FlpOut of R25C01-GAl4 highlights the expression of the two Tier $1 \mathrm{WPN}_{B} S$ (blue) and a Tier 2/3 WPN $\mathrm{B}_{\mathrm{B}}$ (green, arrow). N-Cadherin delineates neuropil (magenta). J, Some ${ }_{W P N}{ }_{B}{ }^{\prime}$ somata (green; R25C01-GAL4) colocalize with ChAT (magenta) Trojan-LeXA::0FAD protein trap but not vGLUT (K; magenta) Trojan-LexA::0FAD protein trap. Scale bars: $\boldsymbol{A}, 25 \mu \mathrm{m} ; \boldsymbol{H}, \boldsymbol{I}$, $50 \mu \mathrm{m} ; J, K, 20 \mu \mathrm{m}$.

\section{Discussion}

Diverse synaptic connectivity and cell class-specific serotonin receptor expression contribute to serotonin's complex effects on sensory processing and behavior. Whole-brain EM datasets (Ohyama et al., 2015; Schlegel et al., 2017; Meinertzhagen, 2018; Bates et al., 2019; Cook et al., 2019) can provide a comprehensive understanding of the synaptic connectivity of neurons across networks, informing predictions about the function of serotonin within discrete networks. Here, we determined the synaptic connectivity of a broadly projecting, identified serotonin neuron within and between olfactory brain regions at a single-cell resolution (Fig. 11). The CSDn illustrates that a single modulatory neuron can follow different connectivity rules between sensory networks (i.e., the AL and the $\mathrm{LH}$ ), suggesting that it is heavily interconnected with local circuits in a nuanced manner, even differentially interacting with cell classes that support distinct local 


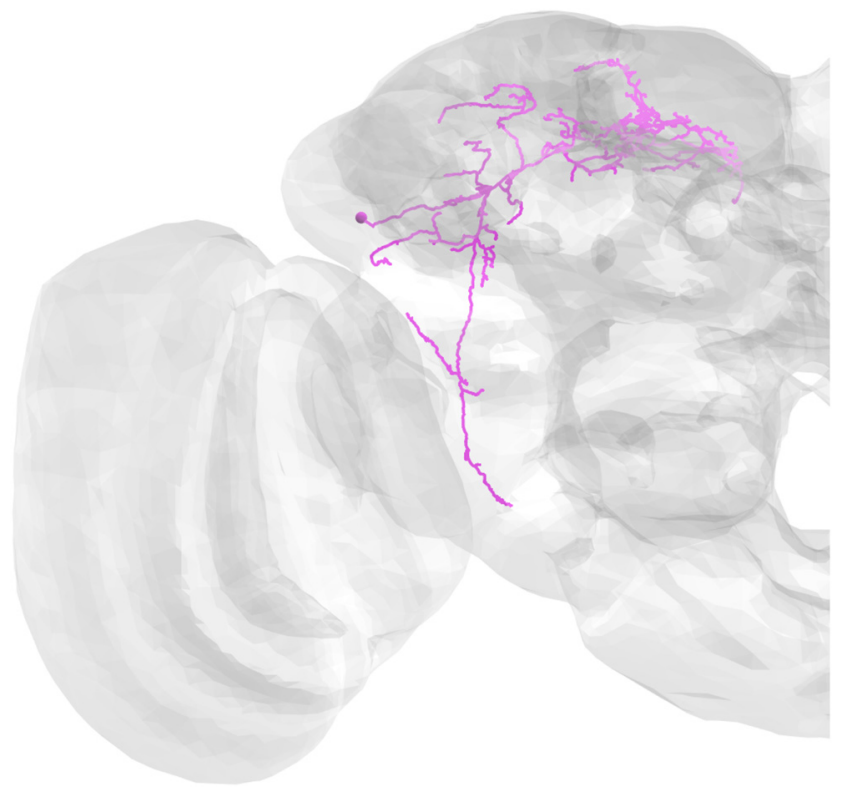

Figure 9. Extrinsic input from a protocerebral neuron. EM reconstruction of a previously undescribed protocerebral neuron that provides strong input (at least 90 synapses) to the CSDns in the superior medial protocerebrum, superior lateral protocerebrum, and antler.

computations. CSDn also receives input at several scales, from diffuse local input, to strong glomerulus-specific input (in the case of the SIMPALs) and global input (in the case of the $\mathrm{WPN}_{\mathrm{B}} \mathrm{s}$ ). Thus, a single modulatory neuron simultaneously integrates input at different scales, likely exerting differential effects on distinct stages of sensory processing.

\section{Distinct connectivity within and across sensory regions}

Within the olfactory system of vertebrate and invertebrates, serotonin can have stimulus-specific effects on odor-evoked responses or affect only a subset of a given neuron class because of polysynaptic consequences of modulation and the diversity of serotonin receptors expressed in olfactory networks (Gaudry, 2018). For instance, serotonin differentially causes direct and polysynaptic excitation of mitral cells in the main olfactory bulb (S. Liu et al., 2012; Brill et al., 2016; Kapoor et al., 2016), yet direct and polysynaptic inhibition of mitral cells in the accessory olfactory bulb (Huang et al., 2017). Furthermore, the combined release of serotonin and glutamate from dorsal raphe neurons differentially affects mitral and tufted cells in the main olfactory bulb, decorrelating odor-evoked responses of mitral cells and enhancing the odor-evoked responses of tufted cells (Kapoor et al., 2016). This could enhance the discriminability of mitral cell odor representations while simultaneously enhancing the sensitivity of tufted cells, allowing simultaneous modulation of different odor representations. We found that the CSDn is differentially connected to distinct LN and PN subtypes (Figs. 3, 4). Moreover, LN and PN subtypes in Drosophila have different serotonin receptor expression patterns (Sizemore and Dacks, 2016). Thus, the combined complexity of cell class-specific receptor expression and heterogeneous synaptic connectivity likely underlie the seemingly variable effects of serotonin on olfactory processing.

Serotonergic neurons may also play different roles across olfactory regions. Raphe stimulation differentially affects odorevoked responses of mitral and tufted cells in the main olfactory bulb (Kapoor et al., 2016) yet only affects spontaneous activity rather than the odor-evoked responses of single units recorded in piriform cortex (Lottem et al., 2016). Single serotonergic neurons can span several olfactory regions (Ren et al., 2019) and thus have the potential to simultaneously, and differentially, modulate these networks. Consistent with this, we found general differences in the connectivity of the CSDn across processing stages. In the $\mathrm{AL}$, the CSDn provides concentrated input to subsets of LNs (Figs. 4, 5); however, CSDn input to LHLNs is far more diffuse (Fig. 7), and the degree to which the CSDn synapses with different populations of PNs also differs between the $\mathrm{AL}$ and LH (Fig. 6). Finally, local synaptic input allows the CSDn to exhibit odor invariant inhibition in the AL, yet odor and regionspecific excitation in the LH (X. Zhang et al., 2019). Together, this suggests that single serotonergic neurons can play different functional roles across processing stages.

\section{Glomerulus-specific connectivity}

Topographical representation of stimulus space (e.g., identity or location) is a fundamental property of sensory systems (Kaas, 1997; Patel et al., 2014; Kaneko and Ye, 2015), and overlaid on these sensory maps are the differential projections of modulatory neurons. The density of serotonergic innervation varies between glomerular layers of the olfactory bulb (Gomez et al., 2005; Gracia-Llanes et al., 2010; Muzerelle et al., 2016), AL glomeruli (Singh et al., 2013), and even glomerular subregions (Sun et al., 1993; Lizbinski et al., 2016). Although CSDn active zone density varies across glomeruli (Fig. 2A) (Coates et al., 2017), the CSDn predominantly targets LNs (Fig. $3 B$ ). As a population, AL LNs express all five serotonin receptors (Sizemore and Dacks, 2016), and concordantly serotonin can be excitatory or inhibitory for different LN types depending on the receptor expressed (Suzuki et al., 2020). Here, we identify the specific LNs with which the CSDn interacts, providing strong unidirectional input to densely branching ABAF LNs and reciprocal connectivity to patchy LNs (Fig. 5C). LNs synapse on many principal neurons within the AL and support a wide range of network computations (Olsen and Wilson, 2008; Root et al., 2008; Yaksi and Wilson, 2010; Hong and Wilson, 2015; Nagel et al., 2015). Differential synaptic connectivity with specific LN subtypes may allow the CSDn to preferentially influence or actively participate in neural computations supported by LN subtypes.

What is the significance of the nonuniform glomerular innervation of the CSDn? The number of CSDn active zones within a glomerulus depends entirely on CSDn cable length (Fig. 2C), and the combination of synaptic inputs to the CSDn from ORNs, PNs, and LNs is heterogeneous across glomeruli (Fig. $3 C$ ). Many odors inhibit the CSDn, with inhibition scaling with the degree to which the AL is activated. Glomerulus-specific differences in input demographics suggest that the CSDn may further integrate local synaptic input within the AL in an odor-specific manner. For instance, $\sim 50 \%$ of the input to the CSDn in VM1 is from ORNs, compared with $\sim 90 \%$ of input in VM3 originating from LNs. Thus, the balance of excitation and inhibition experienced by the CSDn, and therefore the influence of the CSDn on olfactory processing, likely depends in part on the odors that are currently being encountered. Alternatively, variation in the input to the CSDn across glomeruli may serve to provide a broad sampling of network activity that can be superseded in a context-dependent manner, for example, by receiving input from strongly connected partners, such as the SIMPAL neurons or specific LN types. The relative balance of excitatory and inhibitory diffuse synaptic input that the CSDn experiences while these strongly connected partners are silent may therefore establish a baseline 

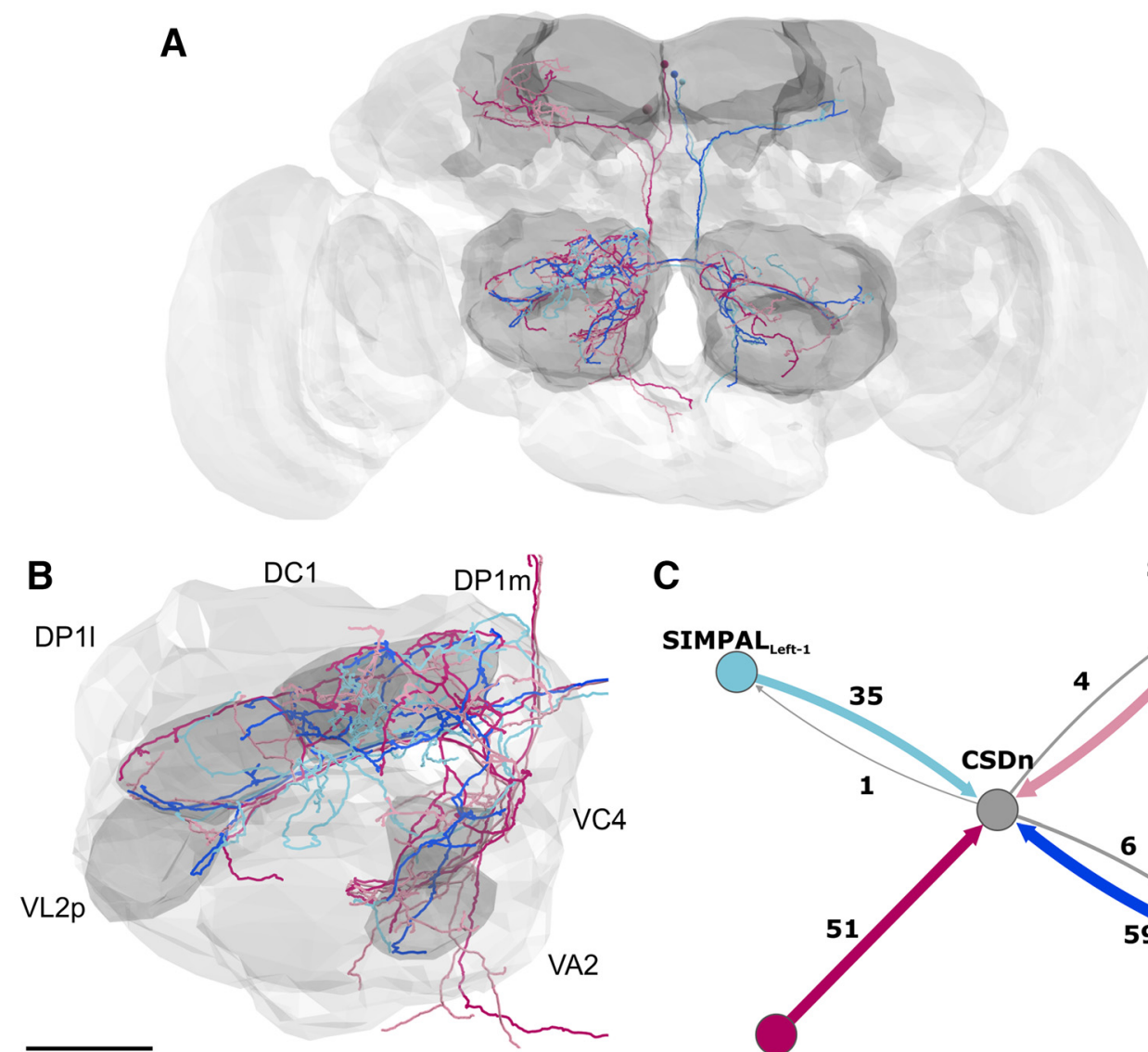

25 um
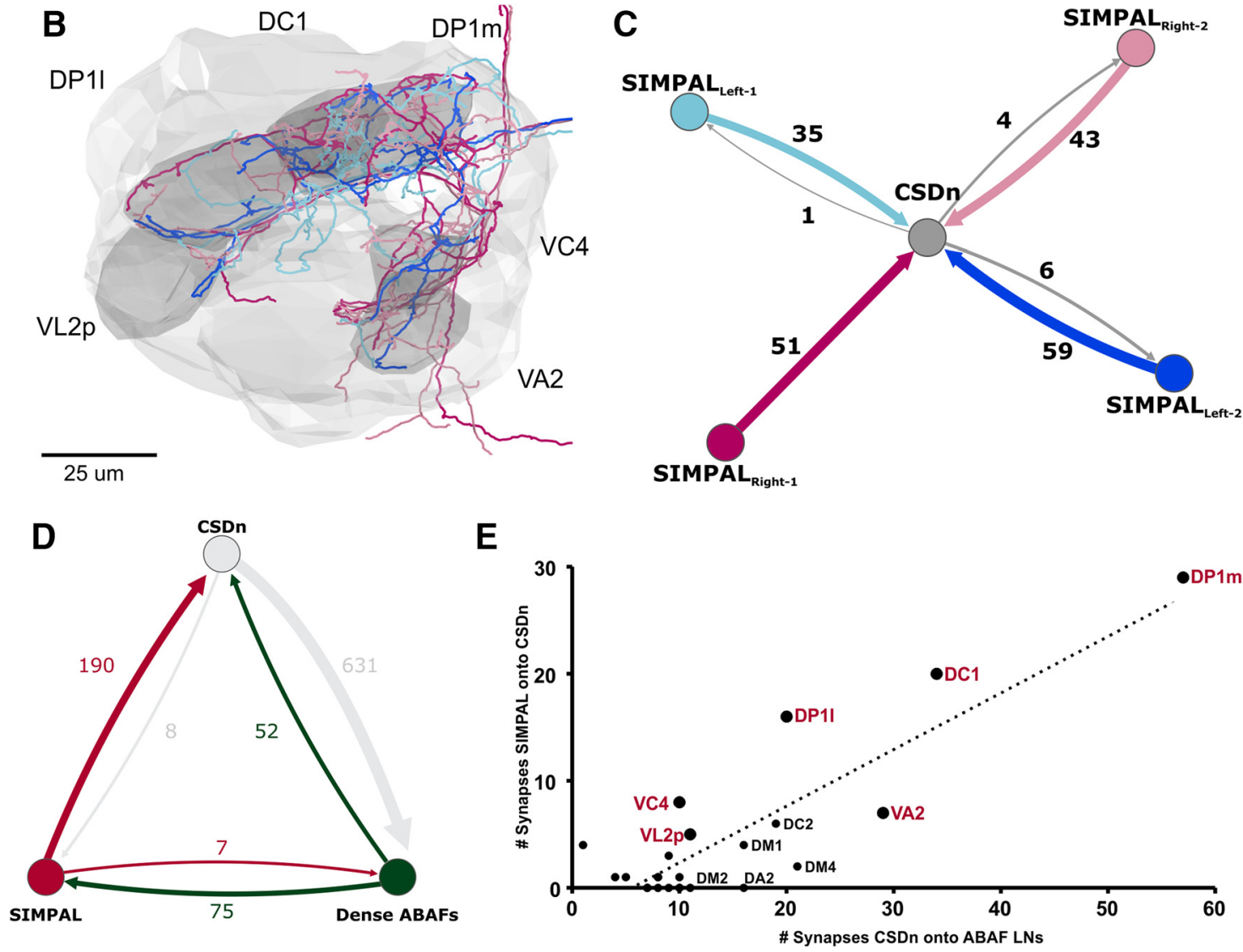

Figure 10. Novel extrinsic input from the SIMPAL neurons. A, EM reconstruction of the four SIMPAL neurons, which provide strong input ( $>10$ synapses) to the CSDns in food odor-associated glomeruli. $\boldsymbol{B}$, DC1, DP1I, DP1m, VA2, VC4, and VL2p. C, Connectivity of individual SIMPAL neurons to the CSDn is predominantly nonreciprocal. D, The CSDns, dense ABAF LNs, and SIMPAL neurons form a feedback loop, suggesting that the CSDn may influence the SIMPAL neurons polysynaptically. $\boldsymbol{E}$, The CSDn provides strong input to the ABAF LNs in the 6 food odor-associated glomeruli in which the SIMPAL neurons synapse on the CSDn.

modulatory tone exerted by the CSDns. Odor-evoked inhibition of the CSDn may therefore represent both direct inhibition of the CSDn and a loss of the diffuse excitatory input that the CSDn receives from cholinergic neurons (e.g., ORNs or PNs) across the AL.

\section{Conservation of connectivity across development,} individuals, and species

There are several contexts in which the connectivity of identified neurons varies. For instance, during metamorphosis, many neurons undergo dramatic changes in morphology and biophysical properties (Consoulas et al., 2000; Duch et al., 2000); thus, these neurons provide the opportunity to determine the extent to which broad principles of connectivity are reconfigured across life stages. Rather than undergoing programmed cell death, the CSDns survive metamorphosis, becoming more elaborated in the AL, and project extensively throughout the protocerebrum (Kent et al., 1987; Roy et al., 2007; Singh et al., 2013). Although many specific synaptic partners of the larval and adult CSDns change, many aspects of the wiring logic are maintained. Similar to adults, larval CSDns receive input from specific ORN types and preferentially target distinct LN subtypes (Berck et al., 2016). However, between life stages, the CSDns receive input from ORNs that express different olfactory receptor proteins and the 
Table 2. Synaptic partners identified across EM datasets ${ }^{a}$

\begin{tabular}{lc}
\hline FAFB neuron & Hemibrain body ID \\
\hline CSDn left hand & 759810119 \\
CSDn right hand & 851459972 \\
Dense ABAF & 5813024698 \\
Dense ABAF & 1640909284 \\
LN $_{\text {SEZ }}$ & 5813018460 \\
LN $_{\text {SEZ }}$ & 5901206553 \\
Patchy LN & 1671257931 \\
Patchy LN & 1704347707 \\
Patchy LN & 1857799548 \\
SIMPAL & 1727975215 \\
SIMPAL & 704699661 \\
SIMPAL & 5812996052 \\
SIMPAL & 693927941 \\
WPN $_{B} 1$ & 5813047683 \\
WPN $_{B} 1$ & 787563949 \\
WPN $_{B} 2 / 3$ & 849279791 \\
\hline
\end{tabular}

${ }^{a}$ Several key synaptic partners that we reconstructed in the FAFB EM dataset were also identified in a second recently released EM volume of a portion of a Drosophila central brain (the "hemibrain" (Scheffer et al., 2020)).

interanimal variability between identified stomatogastric ganglion neurons (Otopalik et al., 2017b) and variations in the morphology of Drosophila visual neurons underlies phenotypic variation in visual-guided behavior (Linneweber et al., 2020). Although we replicated several key synaptic relationships (Table 2) between the CSDns and identified neurons using a second densely reconstructed EM volume of a different fly brain (Scheffer et al., 2020) and synaptic relationships previously reported using transgenic approaches for specific ORN and PN subpopulations (Coates et al., 2017), whether the precise glomerulus-specific synapse fractions for input to the CSDn are consistent across animals is unclear. There is evidence, however, for some degree of interanimal variability in the connectivity of the CSDns. Based on synaptobrevin-linked GRASP, populations of DM1 and VM1 ORNs and DM5 PNs reliably provide synaptic input to the CSDns across animals (Coates et al., 2017) (Fig. 3C). However, some DA2 and VM2 ORNs only synapse on the CSDns in a subset of animals (Coates et al., 2017). Although interanimal variability in connectivity can arise from genetic background and experience, Coates et al. (2017) used flies from a single cohort, so the observed variability in ORN input likely arises because of intrinsic developmental variation. In the AL, the number of ORNs and PNs innervating a given glomerulus varies across animals (Grabe et al., 2016) and even between brain hemispheres (Tobin et al., 2017). Furthermore, individual patchy LNs innervate different sets of glomeruli across animals (Chou et al., 2010), suggesting that these LNs integrate synaptic input from different glomeruli in each animal. Intrinsic developmental variation supports behavioral phenotypic diversity in a nonheritable manner; thus, while many glomerulus-specific synaptic inputs to the CSDns are consistent, the precise demography of these inputs likely varies to some degree across animals.

Finally, the connectivity of homologous neurons also varies across species (Lillvis and Katz, 2013), supporting different functional roles within a given network. For instance, homologous interneurons in nudibranch species with similar swimming behavior have different network connectivity and activity during swimming motor output (Sakurai et al., 2011). The general morphology of CSDns is conserved across most holometabolous insects (Dacks et al., 2006), and EM studies in Manduca sexta demonstrate that, similar to Drosophila, the CSDns in moths receive input from both PNs and LNs (Sun et al., 1993). However, as opposed to Drosophila, recordings from CSDn processes in the AL processes in moths indicate that these neurons are excited by olfactory stimuli (Hill et al., 2002; Zhao and Berg, 2009), demonstrating that the conserva-

CSDns appear to target functionally distinct sets of LNs. Thus, broad principles, such as heterogeneous input and robust connectivity to local inhibitory networks, are conserved, although the precise functional roles played by the strongest synaptic partners may differ.

The morphology, and likely the connectivity, of identified neurons also varies between individual animals (Hiesinger and Hassan, 2018). For example, detailed analyses of branching patterns and cable properties demonstrate a high degree of tion of morphology and transmitter content of the CSDns is not predictive of its function across taxa.

\section{The CSDn as an intrinsic and extrinsic modulatory neuron}

The CSDn receives input both from circuits intrinsic to the AL and $\mathrm{LH}$, and top-down, extrinsic input. This suggests that the CSDns can act as both intrinsic and extrinsic modulatory neurons as their activity reflects ongoing olfactory network history 
and activity in other networks (Katz, 1995; Lizbinski and Dacks, 2018). One source of top-down input to the CSDns are the $\mathrm{WPN}_{\mathrm{B}} \mathrm{S}$, which are weakly activated by lateral wind input (Suver et al., 2019). As a population, WPNs likely encode many features of mechanosensory input, including wind stimuli, which can be integrated with olfactory cues to locate and orient to food and mates. Each $\mathrm{WPN}_{\mathrm{B}}$ tier may therefore provide information about different aspects of ongoing mechanosensory input to the antennae, such as direction, intensity, or vibration frequency. Furthermore, the positioning of their input along the main process, where the CSDn branch diameter is relatively wide (X. Zhang et al., 2019), may allow the $\mathrm{WPN}_{\mathrm{B}}$ s to provide input that can spread to CSDn processes across olfactory neuropils.

The CSDn also receives strong synaptic input within the AL from four protocerebral neurons, the SIMPAL neurons (Fig. 10) in several food-odor associated glomeruli. Although the CSDn has little direct synaptic feedback to the SIMPAL neurons, they likely provide polysynaptic feedback via the ABAF LNs in these food-responsive glomeruli. Nevertheless, the SIMPAL neurons' influence on the CSDn is likely constrained to the AL and tempered by ongoing network dynamics. In contrast, the $\mathrm{WPN}_{\mathrm{B}} \mathrm{S}$ synapse on the CSDns at multiple locations throughout the protocerebrum along the widest CSDn process (X. Zhang et al., 2019), potentially allowing for greater influence over CSDn compartments.

In conclusion, population-level diversity allows serotonergic neurons to influence a diverse range of behaviors, often in a context-dependent manner (Okaty et al., 2019). Serotonergic raphe neurons integrate input from 80 anatomically distinct areas (Ogawa et al., 2014; Pollak Dorocic et al., 2014; Weissbourd et al., 2014), which allow them to respond to immediate stimuli, such as reward, punishment, or both (Ren et al., 2018) or broad physiological states that vary over longer time courses (Jacobs et al., 1981; Monti, 2011). This complex connectivity likely supports the context-dependent effects of the raphe, for instance, evoking escape behaviors under high threat conditions yet reducing movement under low threat conditions (Seo et al., 2019). The functional role of the CSDns is likely even more complex than their connectivity suggests. For instance, somatodendritic and axonal expression of the serotonin reuptake transporter by the CSDns have different effects on odor-guided behavior (Kasture et al., 2019), suggesting that compartment-specific functional heterogeneity. Furthermore, serotonergic neurons can also release serotonin in a paracrine manner (Bunin and Wightman, 1998, 1999) and can coexpress other transmitters (Fu et al., 2010; Z. Liu et al., 2014; Sengupta et al., 2017; Ren et al., 2018; Huang et al., 2019). Thus, diverse properties and complex connectivity likely allow individual serotonergic neurons to have a nuanced influence on behavior.

\section{References}

Ache BW, Young JM (2005) Olfaction: diverse species, conserved principles. Neuron 48:417-430.

Agnati LF, Bjelke B, Fuxe K (1995) Volume versus wiring transmission in the brain: a new theoretical frame for neuropsychopharmacology. Med Res Rev 15:33-45.

Andrade R, Haj-Dahmane S (2013) Serotonin neuron diversity in the dorsal raphe. ACS Chem Neurosci 4:22-25.

Bang SJ, Jensen P, Dymecki SM, Commons KG (2012) Projections and interconnections of genetically defined serotonin neurons in mice. Eur J Neurosci 35:85-96.

Bates AS, Janssens J, Jefferis GS, Aerts S (2019) Neuronal cell types in the fly: single-cell anatomy meets single-cell genomics. Curr Opin Neurobiol $56: 125-134$
Bates AS, Manton JD, Jagannathan SR, Costa M, Schlegel P, Rohlfing T, Jefferis G (2020a) The natverse, a versatile toolbox for combining and analysing neuroanatomical data.Elife 9:e53350.

Bates AS, Schlegel P, Roberts RJ, Drummond N, Tamimi IF, Turnbull R, Zhao X, Marin EC Popovici PD, Dhawan S, Jamasb JA, Li F, Rubin GM, Waddell S, Bock DD, Costa M, Jefferis G (2020b) Complete connectomic reconstruction of olfactory projection neurons in the fly brain. Curr Biol. Advance online publication. Retrieved January 20, 2020. doi: 10.1016/j. cub.2020.06.042.

Berck ME, Khandelwal A, Claus L, Hernandez-Nunez L, Si G, Tabone CJ, Li F, Truman JW, Fetter RD, Louis M, Samuel AD, Cardona A (2016) The wiring diagram of a glomerular olfactory system. Elife 5:e14859.

Brill J, Shao Z, Puche AC, Wachowiak M, Shipley MT (2016) Serotonin increases synaptic activity in olfactory bulb glomeruli. J Neurophysiol 115:1208-1219.

Bunin MA, Wightman RM (1998) Quantitative evaluation of 5-hydroxytryptamine (serotonin) neuronal release and uptake: an investigation of extrasynaptic transmission. J Neurosci 18:4854-4860.

Bunin MA, Wightman RM (1999) Paracrine neurotransmission in the CNS: involvement of 5-HT. Trends Neurosci 22:377-382.

Carlsson MA, Diesner M, Schachtner J, Nässel DR (2010) Multiple neuropeptides in the Drosophila antennal lobe suggest complex modulatory circuits. J Comp Neurol 518:3359-3380.

Caron SJ, Ruta V, Abbott LF, Axel R (2013) Random convergence of olfactory inputs in the Drosophila mushroom body. Nature 497:113-117.

Chiang AS, Lin CY, Chuang CC, Chang HM, Hsieh CH, Yeh CW, Shih CT, Wu JJ, Wang GT, Chen YC, Wu CC, Chen GY, Ching YT, Lee PC, Lin CY, Lin HH, Wu CC, Hsu HW, Huang YA, Chen JY, et al. (2011) Threedimensional reconstruction of brain-wide wiring networks in Drosophila at single-cell resolution. Curr Biol 21:1-11.

Chou YH, Spletter ML, Yaksi E, Leong JC, Wilson RI, Luo L (2010) Diversity and wiring variability of olfactory local interneurons in the Drosophila antennal lobe. Nat Neurosci 13:439-449.

Coates KE, Majot AT, Zhang X, Michael CT, Spitzer SL, Gaudry Q, Dacks AM (2017) Identified serotonergic modulatory neurons have heterogeneous synaptic connectivity within the olfactory system of Drosophila. J Neurosci 37:7318-7331.

Consoulas C, Duch C, Bayline RJ, Levine RB (2000) Behavioral transformations during metamorphosis: remodeling of neural and motor systems. Brain Res Bull 53:571-583.

Cook SJ, Jarrell TA, Brittin CA, Wang Y, Bloniarz AE, Yakovlev MA, Nguyen KC, Tang LT, Bayer EA, Duerr JS, Bulow HE, Hobert O, Hall DH, Emmons SW (2019) Whole-animal connectomes of both Caenorhabditis elegans sexes. Nature 571:63-71.

Costa M, Manton JD, Ostrovsky AD, Prohaska S, Jefferis GS (2016) NBLAST: rapid, sensitive comparison of neuronal structure and construction of neuron family databases. Neuron 91:293-311.

Dacks AM, Christensen TA, Hildebrand JG (2006) Phylogeny of a serotoninimmunoreactive neuron in the primary olfactory center of the insect brain. J Comp Neurol 498:727-746.

Das A, Chiang A, Davla S, Priya R, Reichert H, Vijayraghavan K, Rodrigues V (2011) Identification and analysis of a glutamatergic local interneuron lineage in the adult Drosophila olfactory system. Neural Syst Circuits 1:4.

Diao F, Ironfield H, Luan H, Diao F, Shropshire WC, Ewer J, Marr E, Potter CJ, Landgraf M, White BH (2015) Plug-and-play genetic access to Drosophila cell types using exchangeable exon cassettes. Cell Rep 10:1410-1421.

Dolan MJ, Frechter S, Bates AS, Dan C, Huoviala P, Roberts RJ, Schlegel P, Dhawan S, Tabano R, Dionne H, Christoforou C, Close K, Sutcliffe B, Giuliani B, Li F, Costa M, Ihrke G, Meissner GW, Bock DD, Aso Y, et al. (2019) Neurogenetic dissection of the Drosophila lateral horn reveals major outputs, diverse behavioural functions, and interactions with the mushroom body. Elife 8:e43079.

Duch C, Bayline RJ, Levine RB (2000) Postembryonic development of the dorsal longitudinal flight muscle and its innervation in Manduca sexta. J Comp Neurol 422:1-17.

Dweck HK, Ebrahim SA, Kromann S, Bown D, Hillbur Y, Sachse S, Hansson BS, Stensmyr MC (2013) Olfactory preference for egg laying on citrus substrates in Drosophila. Curr Biol 23:2472-2480.

Eid L, Champigny MF, Parent A, Parent M (2013) Quantitative and ultrastructural study of serotonin innervation of the globus pallidus in squirrel monkeys. Eur J Neurosci 37:1659-1668. 
Fouquet W, Owald D, Wichmann C, Mertel S, Depner H, Dyba M, Hallermann S, Kittel RJ, Eimer S, Sigrist SJ (2009) Maturation of active zone assembly by Drosophila Bruchpilot. J Cell Biol 186:129-145.

Frechter S, Bates AS, Tootoonian S, Dolan MJ, Manton J, Jamasb AR, Kohl J, Bock D, Jefferis G (2019) Functional and anatomical specificity in a higher olfactory centre. Elife 8:e44590.

Fu W, Le Maître E, Fabre V, Bernard JF, Xu ZQ, Hökfelt T (2010) Chemical neuroanatomy of the dorsal raphe nucleus and adjacent structures of the mouse brain. J Comp Neurol 518:3464-3494.

Fuxe K, Agnati LF, Marcoli M, Borroto-Escuela DO (2015) Volume transmission in central dopamine and noradrenaline neurons and its astroglial targets. Neurochem Res 40:2600-2614.

Fuyama Y (1976) Behavior genetics of olfactory responses in Drosophila: I. Olfactometry and strain differences in Drosophila melanogaster. Behav Genet 6:407-420.

Gaudry Q (2018) Serotonergic modulation of olfaction in rodents and insects. Yale J Biol Med 91:23-32.

Gomez C, Brinon JG, Barbado MV, Weruaga E, Valero J, Alonso JR (2005) Heterogeneous targeting of centrifugal inputs to the glomerular layer of the main olfactory bulb. J Chem Neuroanat 29:238-254.

Grabe V, Sachse S (2018) Fundamental principles of the olfactory code. BioSystems 164:94-101.

Grabe V, Baschwitz A, Dweck HK, Lavista-Llanos S, Hansson BS, Sachse S (2016) Elucidating the neuronal architecture of olfactory glomeruli in the Drosophila antennal lobe. Cell Rep 16:3401-3413.

Gracia-Llanes FJ, Blasco-Ibáñez JM, Nácher J, Varea E, Liberia T, Martínez P, Martínez-Guijarro FJ, Crespo C (2010) Synaptic connectivity of serotonergic axons in the olfactory glomeruli of the rat olfactory bulb. Neuroscience 169:770-780.

Groschner LN, Miesenbock G (2019) Mechanisms of sensory discrimination: insights from Drosophila olfaction. Annu Rev Biophys 48:209-229.

Halliday GM, Li YW, Joh TH, Cotton RG, Howe PR, Geffen LB, Blessing WW (1988) Distribution of monoamine-synthesizing neurons in the human medulla oblongata. J Comp Neurol 273:301-317.

Hiesinger PR, Hassan BA (2018) The evolution of variability and robustness in neural development. Trends Neurosci 41:577-586.

Hill ES, Iwano M, Gatellier L, Kanzaki R (2002) Morphology and physiology of the serotonin-immunoreactive putative antennal lobe feedback neuron in the male silkmoth Bombyx mori. Chem Senses 27:475-483.

Hong EJ, Wilson RI (2015) Simultaneous encoding of odors by channels with diverse sensitivity to inhibition. Neuron 85:573-589.

Huang KW, Ochandarena NE, Philson AC, Hyun M, Birnbaum JE, Cicconet M, Sabatini BL (2019) Molecular and anatomical organization of the dorsal raphe nucleus. Elife 8:e46464.

Huang Z, Thiebaud N, Fadool DA (2017) Differential serotonergic modulation across the main and accessory olfactory bulbs. J Physiol 595:35153533.

Huoviala P, Dolan MJ, Love FM, Frechter S, Roberts RJ, Mitrevica Z, Schlegel P, Bates AS, Aso Y, Rodrigues T, Cornwall H, Stensmyr M, Bock D, Rubin GM, Costa M, Jefferis G (2019) Neural circuit basis of aversive odour processing in Drosophila from sensory input to descending output. BioRxiv 394403. doi: 10.1101/394403.

Ignell R, Root CM, Birse RT, Wang JW, Nässel DR, Winther AM (2009) Presynaptic peptidergic modulation of olfactory receptor neurons in Drosophila. Proc Natl Acad Sci USA 106:13070-13075.

Ishimura K, Takeuchi Y, Fujiwara K, Tominaga M, Yoshioka H, Sawada T (1988) Quantitative analysis of the distribution of serotonin-immunoreactive cell bodies in the mouse brain. Neurosci Lett 91:265-270.

Ito M, Masuda N, Shinomiya K, Endo K, Ito K (2013) Systematic analysis of neural projections reveals clonal composition of the Drosophila brain. Curr Biol 23:644-655.

Jacobs BL, Heym J, Trulson ME (1981) Behavioral and physiological correlates of brain serotoninergic unit activity. J Physiol (Paris) 77:431-436.

Jeanne JM, Wilson RI (2015) Convergence, divergence, and reconvergence in a feedforward network improves neural speed and accuracy. Neuron 88:1014-1026.

Jeanne JM, Fiș ek M, Wilson RI (2018) The organization of projections from olfactory glomeruli onto higher-order neurons. Neuron 98:1198-1213.e6.

Jensen P, Farago AF, Awatramani RB, Scott MM, Deneris ES, Dymecki SM (2008) Redefining the serotonergic system by genetic lineage. Nat Neurosci 11:417-419.
Kaas JH (1997) Topographic maps are fundamental to sensory processing. Brain Res Bull 44:107-112.

Kamikouchi A, Inagaki HK, Effertz T, Hendrich O, Fiala A, Gopfert MC, Ito K (2009) The neural basis of Drosophila gravity-sensing and hearing. Nature 458:165-171.

Kaneko T, Ye B (2015) Fine-scale topography in sensory systems: insights from Drosophila and vertebrates. J Comp Physiol A Neuroethol Sens Neural Behav Physiol 201:911-920.

Kapoor V, Provost AC, Agarwal P, Murthy VN (2016) Activation of raphe nuclei triggers rapid and distinct effects on parallel olfactory bulb output channels. Nat Neurosci 19:271-282.

Kasture AS, Bartel D, Steinkellner T, Sucic S, Hummel T, Freissmuth M (2019) Distinct contribution of axonal and somatodendritic serotonin transporters in Drosophila olfaction. Neuropharmacology 161:107564.

Katz PS (1995) Intrinsic and extrinsic neuromodulation of motor circuits. Curr Opin Neurobiol 5:799-808.

Kent KS, Hoskins SG, Hildebrand JG (1987) A novel serotonin-immunoreactive neuron in the antennal lobe of the sphinx moth Manduca sexta persists throughout postembryonic life. J Neurobiol 18:451-465.

Knaden M, Strutz A, Ahsan J, Sachse S, Hansson BS (2012) Spatial representation of odorant valence in an insect brain. Cell Rep 1:392-399.

Lai JS, Lo SJ, Dickson BJ, Chiang AS (2012) Auditory circuit in the Drosophila brain. Proc Natl Acad Sci USA 109:2607-2612.

Li PH, Linsdsey LF, Januszewski M, Zheng Z, Bates AS, Taisz I, Tyka M, Nichols M, Li F, Perlman E, Maitin-Shepard J, Blakely T, Leavitt L, Jefferis G, Bock D, Jain V (2019) Automated reconstruction of a serialsection EM Drosophila brain with flood-filling networks and local realignment. BioRxiv 605634. doi: 10.1101/605634.

Liang L, Li Y, Potter CJ, Yizhar O, Deisseroth K, Tsien RW, Luo L (2013) GABAergic projection neurons route selective olfactory inputs to specific higher-order neurons. Neuron 79:917-931.

Lillvis JL, Katz PS (2013) Parallel evolution of serotonergic neuromodulation underlies independent evolution of rhythmic motor behavior. J Neurosci 33:2709-2717.

Linneweber GA, Andriatsilavo M, Dutta SB, Bengochea M, Hellbruegge L, Liu G, Ejsmont RK, Straw AD, Wernet M, Hiesinger PR, Hassan BA (2020) A neurodevelopmental origin of behavioral individuality in the Drosophila visual system. Science 367:1112-1119.

Liu S, Aungst JL, Puche AC, Shipley MT (2012) Serotonin modulates the population activity profile of olfactory bulb external tufted cells. J Neurophysiol 107:473-483.

Liu WW, Wilson RI (2013) Glutamate is an inhibitory neurotransmitter in the Drosophila olfactory system. Proc Natl Acad Sci USA 110:1029410299

Liu Z, Zhou J, Li Y, Hu F, Lu Y, Ma M, Feng Q, Zhang JE, Wang D, Zeng J, Bao J, Kim JY, Chen ZF, El Mestikawy S, Luo M (2014) Dorsal raphe neurons signal reward through 5-HT and glutamate. Neuron 81:1360-1374.

Lizbinski KM, Dacks AM (2018) Intrinsic and extrinsic neuromodulation of olfactory processing. Front Cell Neurosci 11:424.

Lizbinski KM, Metheny JD, Bradley SP, Kesari A, Dacks AM (2016) The anatomical basis for modulatory convergence in the antennal lobe of Manduca sexta. J Comp Neurol 524:1859-1875.

Lottem E, Lorincz ML, Mainen ZF (2016) Optogenetic activation of dorsal raphe serotonin neurons rapidly inhibits spontaneous but not odorevoked activity in olfactory cortex. J Neurosci 36:7-18

MacQueen B (1967) Some methods for classification and analysis of multivariate observations. Proceedings of 5th Berkeley Symposium on Mathematical Statistics and Probability 1:281-297.

Marder E (2012) Neuromodulation of neuronal circuits: back to the future. Neuron 76:1-11.

Marin EC, Roberts RJ, Büld L, Theiss M, Pleijzier MW, Sarkissian T, Laursen WJ, Turnbull R, Schlegel P, Bates AS, Li F, Landgraf M, Costa M, Bock DD, Garrity PA, Jefferis G (2020) Connectomics analysis reveals first, second, and third order thermosensory and hygrosensory neurons in the adult Drosophila brain. Curr Biol. Advance online Publication. Retrieved July 2, 2020. doi: 10.1016/j.cub.2020.06.028.

Matsuo E, Seki H, Asai T, Morimoto T, Miyakawa H, Ito K, Kamikouchi A (2016) Organization of projection neurons and local neurons of the primary auditory center in the fruit fly Drosophila melanogaster. J Comp Neurol 524:1099-1164.

Meinertzhagen IA (2018) Of what use is connectomics? A personal perspective on the Drosophila connectome. J Exp Biol 221:jeb164954. 
Milyaev N, Osumi-Sutherland D, Reeve S, Burton N, Baldock RA, Armstrong JD (2012) The Virtual Fly Brain browser and query interface. Bioinformatics 28:411-415.

Monti JM (2011) Serotonin control of sleep-wake behavior. Sleep Med Rev $15: 269-281$.

Morales M, Margolis EB (2017) Ventral tegmental area: cellular heterogeneity, connectivity and behaviour. Nat Rev Neurosci 18:73-85.

Mosca TJ, Luo L (2014) Synaptic organization of the Drosophila antennal lobe and its regulation by the Teneurins. Elife 3:e03726.

Muzerelle A, Scotto-Lomassese S, Bernard JF, Soiza-Reilly M, Gaspar P (2016) Conditional anterograde tracing reveals distinct targeting of individual serotonin cell groups (B5-B9) to the forebrain and brainstem. Brain Struct Funct 221:535-561.

Nadim F, Bucher D (2014) Neuromodulation of neurons and synapses. Curr Opin Neurobiol 29:48-56

Nagel KI, Hong EJ, Wilson RI (2015) Synaptic and circuit mechanisms promoting broadband transmission of olfactory stimulus dynamics. Nat Neurosci 18:56-65.

Nern A, Pfeiffer BD, Rubin GM (2015) Optimized tools for multicolor stochastic labeling reveal diverse stereotyped cell arrangements in the fly visual system. Proc Natl Acad Sci USA 112:2967-2976.

O'Hearn E, Molliver ME (1984) Organization of raphe-cortical projections in rat: a quantitative retrograde study. Brain Res Bull 13:709-726.

Ogawa SK, Cohen JY, Hwang D, Uchida N, Watabe-Uchida M (2014) Organization of monosynaptic inputs to the serotonin and dopamine neuromodulatory systems. Cell Rep 8:1105-1118.

Ohyama T, Schneider-Mizell CM, Fetter RD, Aleman JV, Franconville R, Rivera-Alba M, Mensh BD, Branson KM, Simpson JH, Truman JW, Cardona A, Zlatic M (2015) A multilevel multimodal circuit enhances action selection in Drosophila. Nature 520:633-639.

Okaty BW, Freret ME, Rood BD, Brust RD, Hennessy ML, deBairos D, Kim JC, Cook MN, Dymecki SM (2015) Multi-scale molecular deconstruction of the serotonin neuron system. Neuron 88:774-791.

Okaty BW, Commons KG, Dymecki SM (2019) Embracing diversity in the 5-HT neuronal system. Nat Rev Neurosci 20:397-424.

Olsen SR, Wilson RI (2008) Lateral presynaptic inhibition mediates gain control in an olfactory circuit. Nature 452:956-960.

Olsen SR, Bhandawat V, Wilson RI (2010) Divisive normalization in olfactory population codes. Neuron 66:287-299.

Otopalik AG, Sutton AC, Banghart M, Marder E (2017a) When complex neuronal structures may not matter. Elife 6:e23508.

Otopalik AG, Goeritz ML, Sutton AC, Brookings T, Guerini C, Marder E (2017b) Sloppy morphological tuning in identified neurons of the crustacean stomatogastric ganglion. Elife 6:e22352.

Owald D, Fouquet W, Schmidt M, Wichmann C, Mertel S, Depner H, Christiansen F, Zube C, Quentin C, Korner J, Urlaub H, Mechtler K, Sigrist SJ (2010) A Syd-1 homologue regulates presynaptic and postsynaptic maturation in Drosophila. J Cell Biol 188:565-579.

Parnas M, Lin AC, Huetteroth W, Miesenbock G (2013) Odor discrimination in Drosophila: from neural population codes to behavior. Neuron 79:932-944.

Patel GH, Kaplan DM, Snyder LH (2014) Topographic organization in the brain: searching for general principles. Trends Cogn Sci 18:351-363.

Patella P, Wilson RI (2018) Functional maps of mechanosensory features in the Drosophila brain. Curr Biol 28:1189-1203.e5.

Pollak Dorocic I, Furth D, Xuan Y, Johansson Y, Pozzi L, Silberberg G, Carlen M, Meletis K (2014) A whole-brain atlas of inputs to serotonergic neurons of the dorsal and median raphe nuclei. Neuron 83:663-678.

Prieto-Godino LL, Rytz R, Cruchet S, Bargeton B, Abuin L, Silbering AF, Ruta V, Dal Peraro M, Benton R (2017) Evolution of acid-sensing olfactory circuits in drosophilids. Neuron 93:661-676.e6.

Ren J, Friedmann D, Xiong J, Liu CD, Ferguson BR, Weerakkody T, DeLoach KE, Ran C, Pun A, Sun Y, Weissbourd B, Neve RL, Huguenard J, Horowitz MA, Luo L (2018) Anatomically defined and functionally distinct dorsal raphe serotonin sub-systems. Cell 175:472-487.e20.

Ren J, Isakova A, Friedmann D, Zeng J, Grutzner SM, Pun A, Zhao GQ, Kolluru SS, Wang R, Lin R, Li P, Li A, Raymond JL, Luo Q, Luo M, Quake SR, Luo L (2019) Single-cell transcriptomes and whole-brain projections of serotonin neurons in the mouse dorsal and median raphe nuclei. Elife 8:e49424.
Root CM, Masuyama K, Green DS, Enell LE, Nässel DR, Lee CH, Wang JW (2008) A presynaptic gain control mechanism fine-tunes olfactory behavior. Neuron 59:311-321.

Rousseeuw P (1987) Silhouettes: a graphical aid to the interpretation and validation of cluster analysis. Comput Appl Math 20:53-65.

Roy B, Singh AP, Shetty C, Chaudhary V, North A, Landgraf M, Vijayraghavan K, Rodrigues V (2007) Metamorphosis of an identified serotonergic neuron in the Drosophila olfactory system. Neural Dev 2:20.

Saalfeld S, Cardona A, Hartenstein V, Tomancak P (2009) CATMAID: collaborative annotation toolkit for massive amounts of image data. Bioinformatics 25:1984-1986.

Sakurai A, Newcomb JM, Lillvis JL, Katz PS (2011) Different roles for homologous interneurons in species exhibiting similar rhythmic behaviors. Curr Biol 21:1036-1043.

Scheffer L, Xu C, Januszewski M, Lu Z, Takemura S, Hayworth K, Huang G, Shinomiya K, Maitlin-Shepard J, Berg S, Clements J, Hubbard P, Katz W, Umayam L, Zhao T, Ackerman D, Blakely T, Bogovic J, Dolafi T, Kainmueller D, et al. (2020) A connectome of the adult drosophila central brain. bioRxiv 030213. doi: 10.1101/2020.04.07.030213.

Schlegel P, Costa M, Jefferis GS (2017) Learning from connectomics on the fly. Curr Opin Insect Sci 24:96-105.

Schmid A, Hallermann S, Kittel RJ, Khorramshahi O, Frolich AM, Quentin C, Rasse TM, Mertel S, Heckmann M, Sigrist SJ (2008) Activity-dependent site-specific changes of glutamate receptor composition in vivo. Nat Neurosci 11:659-666.

Schneider-Mizell CM, Gerhard S, Longair M, Kazimiers T, Li F, Zwart MF, Champion A, Midgley FM, Fetter RD, Saalfeld S, Cardona A (2016) Quantitative neuroanatomy for connectomics in Drosophila. Elife 5: e12059.

Schwarz LA, Luo L (2015) Organization of the locus coeruleus-norepinephrine system. Curr Biol 25:R1051-R1056.

Seki Y, Rybak J, Wicher D, Sachse S, Hansson BS (2010) Physiological and morphological characterization of local interneurons in the Drosophila antennal lobe. J Neurophysiol 104:1007-1019.

Semmelhack JL, Wang JW (2009) Select Drosophila glomeruli mediate innate olfactory attraction and aversion. Nature 459:218-223.

Sengupta A, Bocchio M, Bannerman DM, Sharp T, Capogna M (2017) Control of amygdala circuits by $5-\mathrm{HT}$ neurons via $5-\mathrm{HT}$ and glutamate cotransmission. J Neurosci 37:1785-1796.

Seo C, Guru A, Jin M, Ito B, Sleezer BJ, Ho YY, Wang E, Boada C, Krupa NA, Kullakanda DS, Shen CX, Warden MR (2019) Intense threat switches dorsal raphe serotonin neurons to a paradoxical operational mode. Science 363:538-542

Silbering AF, Rytz R, Grosjean Y, Abuin L, Ramdya P, Jefferis GS, Benton R (2011) Complementary function and integrated wiring of the evolutionarily distinct Drosophila olfactory subsystems. J Neurosci 31:1335713375.

Singh AP, Das RN, Rao G, Aggarwal A, Diegelmann S, Evers JF, Karandikar H, Landgraf M, Rodrigues V, Vijayraghavan K (2013) Sensory neuronderived Eph regulates glomerular arbors and modulatory function of a central serotonergic neuron. PLoS Genet 9:e1003452.

Sizemore TR, Dacks AM (2016) Serotonergic modulation differentially targets distinct network elements within the antennal lobe of Drosophila melanogaster. Sci Rep 6:37119.

Sizemore TR, Hurley LM, Dacks A (2020) Serotonergic modulation across sensory modalities. J Neurophysiol 123:2406-2425.

Strutz A, Soelter J, Baschwitz A, Farhan A, Grabe V, Rybak J, Knaden M, Schmuker M, Hansson BS, Sachse S (2014) Decoding odor quality and intensity in the Drosophila brain. Elife 3:e04147.

Sun XJ, Tolbert LP, Hildebrand JG (1993) Ramification pattern and ultrastructural characteristics of the serotonin-immunoreactive neuron in the antennal lobe of the moth Manduca sexta: a laser scanning confocal and electron microscopic study. J Comp Neurol 338:5-16.

Suver MP, Matheson AM, Sarkar S, Damiata M, Schoppik D, Nagel KI (2019) Encoding of wind direction by central neurons in Drosophila. Neuron 102:828-842.e7.

Suzuki Y, Kiyokage E, Sohn J, Hioki H, Toida K (2015) Structural basis for serotonergic regulation of neural circuits in the mouse olfactory bulb. J Comp Neurol 523:262-280.

Suzuki Y, Schenk JE, Tan H, Gaudry Q (2020) A population of interneurons signals changes in the basal concentration of serotonin and mediates gain control in the Drosophila antennal lobe. Curr Biol 30:1110-1118.e4. 
Tobin WF, Wilson RI, Lee WA (2017) Wiring variations that enable and constrain neural computation in a sensory microcircuit. Elife 6:e24838.

Vaughan AG, Zhou C, Manoli DS, Baker BS (2014) Neural pathways for the detection and discrimination of conspecific song in D. melanogaster. Curr Biol 24:1039-1049.

Weissbourd B, Ren J, DeLoach KE, Guenthner CJ, Miyamichi K, Luo L (2014) Presynaptic partners of dorsal raphe serotonergic and GABAergic neurons. Neuron 83:645-662.

Wilson RI (2013) Early olfactory processing in Drosophila: mechanisms and principles. Annu Rev Neurosci 36:217-241.

Wilson RI, Laurent G (2005) Role of GABAergic inhibition in shaping odorevoked spatiotemporal patterns in the Drosophila antennal lobe. J Neurosci 25:9069-9079.

Wylie CJ, Hendricks TJ, Zhang B, Wang L, Lu P, Leahy P, Fox S, Maeno H, Deneris ES (2010) Distinct transcriptomes define rostral and caudal serotonin neurons. J Neurosci 30:670-684.

Yaksi E, Wilson RI (2010) Electrical coupling between olfactory glomeruli. Neuron 67:1034-1047.
Zhang B, Freeman MR, Waddell S (2010) Drosophila neurobiology: a laboratory manual. Cold Spring Harbor, NY: Cold Spring Harbor Laboratory.

Zhang X, Gaudry Q (2016) Functional integration of a serotonergic neuron in the Drosophila antennal lobe. Elife 5:e16836.

Zhang X, Coates K, Dacks A, Gunay C, Lauritzen JS, Li F, Calle-Schuler SA, Bock D, Gaudry Q (2019) Local synaptic inputs support opposing, network-specific odor representations in a widely projecting modulatory neuron. Elife 8:e46839.

Zhao XC, Berg BG (2009) Morphological and physiological characteristics of the serotonin-immunoreactive neuron in the antennal lobe of the male oriental tobacco budworm, Helicoverpa assulta. Chem Senses 34:363372.

Zheng Z, Lauritzen JS, Perlman E, Robinson CG, Nichols M, Milkie D, Torrens O, Price J, Fisher CB, Sharifi N, Calle-Schuler SA, Kmecova L, Ali IJ, Karsh B, Trautman ET, Bogovic JA, Hanslovsky P, Jefferis GS, Kazhdan M, Khairy K, et al. (2018) A complete electron microscopy volume of the brain of adult Drosophila melanogaster. Cell 174:730-743.e22. 